\title{
Hermeneutik sozialer Systeme
}

\section{Konvergenzen zwischen Systemtheorie und philosophischer Hermeneutik}

\author{
Wolfgang Ludwig Schneider \\ Justus-Liebig-Universität, Institut für Soziologie, Karl-Glöcknerstr. 21E, D-6300 Gießen
}

Zusammenfassung: Hermeneutik und Systemtheorie werden in der theoretischen Diskussion typisch als gegenläufige Ansätze gehandelt. Diese Einordnung gründet vor allem auf der Annahme, daß hermeneutisches Verstehen an den Nachvollzug der subjektiven Intentionen von Autoren bzw. Akteuren gebunden sei. Der Beitrag weist nach, daß diese Unterstellung für die philosophische Hermeneutik Gadamers nicht zutrifft. Gadamer konzipiert den Sinn von Texten und Handlungen aus der Perspektive des Interpreten. Der Sinn von Texten und Handlungen erhält dadurch Selbständigkeit gegenüber den Sinnintentionen ihrer Urheber. Der Vorverständnishorizont des Interpreten wird zum zentralen Faktor für die Konstitution von Sinn. Diese Schwerpunktverlagerung im Prozeß der Sinnkonstitution findet ihre Parallele im Verstehens- und Kommunikationsbegriff der Luhmannschen Systemtheorie. Die hier ansetzende Analyse der Begriffe Verstehen und Kommunikation deckt weitreichende Entsprechungen auf zwischen Gadamers Hermeneutik und der Systemtheorie Luhmanns.

\section{Vorbemerkung1}

In der soziologischen und sozialphilosophischen Diskussion der vergangenen Jahrzehnte traten Hermeneutik und Systemtheorie meist als Gegensatzpaar in Erscheinung. Wie in solchen Konstellationen typisch, hat es nicht an Versuchen gefehlt, die eine Seite durch die andere zu ersetzen oder wie von Habermas vorgeführt - die Komplementarität beider ,Paradigmen' zugleich mit der konstitutionslogischen Priorität der einen Seite zu statuieren. Diese Konstellation verliert ihre Plausibilität, nachdem Luhmanns funktional-strukturelle Systemtheorie Kommunikation als basalen Operationsmodus sozialer Systeme bestimmt und Verstehen als konstitutivem Moment von Kommunikation eine besondere Bedeutung zugewiesen hat. Wie Hermeneutik und Systemtheorie voneinander zu unterscheiden sind, wird unter diesen Prämissen auf neue Weise akut. Zu klären ist, wie Hermeneutik und Systemtheorie Verstehen verstehen.

Einen Beitrag zur Beantwortung dieser Frage haben Georg Kneer und Armin Nassehi (1991) in dieser Zeitschrift geliefert. Die Autoren argumentieren dabei aus systemtheoretischer Perspektive und mit dem Anspruch einer systemtheoretischen Revision der Hermeneutik. Die folgenden Überlegungen nehmen ihren Ausgang von der Hermeneutik. Ihr Ziel ist es nicht, dem Übernah-

1 Für ebenso ausführliche wie hilfreiche Diskussion und Kritik danke ich meinen Kollegen Peter Fuchs und Kay Junge. meangebot von Kneer und Nassehi ein anderes entgegenzusetzen. Das würde nur die alte Diskussionskonstellation fortsetzen. Interessanter erscheint es, die Möglichkeit ins Auge zu fassen, daß die üblichen Unvereinbarkeitsunterstellungen obsolet geworden sein könnten, und dies noch viel weitergehend, als bei Kneer und Nassehi bereits angedeutet.

Um diese Vermutung zu substantiieren, soll im folgenden gezeigt werden, daß der als spezifisch systemtheoretisch geltende anti-handlungstheoretische Impetus in der philosophischen Hermeneutik Gadamers auf Parallelen trifft, die sich bis hinein in unerwartete konzeptuelle Übereinstimmungen nachzeichnen lassen. Ihre Beziehung zum systemtheoretischen Konzept von Verstehen und Kommunikation wird dazu ausführlich zu prüfen sein. ${ }^{2}$

2 Aus Raumgründen völlig unberücksichtigt bleiben müssen leider Konvergenzen, die sich im Bereich der sozialwissenschaftlichen Hermeneutikdiskussion mittlerweile feststellen lassen. Vor allem die von Oevermann (vgl. bes. 1979 und 1986) vertretene Methodologie einer "objektiven Hermeneutik" ist hier zu erwähnen. Deren Vorgehensweise, die strikt sequenziell angelegt ist und auf die Explikation symbolisch objektivierter Bedeutungsstrukturen zielt, trifft sich mit der Anlage des systemtheoretischen Kommunikationsbegriffs und dessen scharfer Unterscheidung zwischen kommunikativ generiertem und psychisch prozessiertem Sinn. Was für die Systemtheorie hier zu gewinnen sein könnte, wäre eine präzise auf die Struktur von Kommunikation zugeschnittene Methode, die bis auf die Ebene der Einzelfallanalyse 


\section{Verstehen in der Systemtheorie}

Luhmann konzipiert Systeme als Beobachter. Durch ihr Beobachten legen sie eine Grenze als Differenz zwischen Beobachter und Beobachtetem. Sie differenzieren sich gegen ihre Umwelt, indem sie jeweils mit Bezug auf die Ergebnisse eigener anderer Beobachtungen operieren (vgl. Luhmann 1990b: 29). Verstehen ist eine spezifische Form des Beobachtens. Vorab zu klären ist daher die Struktur von Beobachtungsoperationen.

Beobachten heißt zunächst bezeichnen. Jedes Bezeichnen aber, indem es etwas als etwas bestimmt, unterscheidet das so Bestimmte von anderem (z. B. diesen Stuhl von jenem, von diesem Tisch oder vor allem, was sonst noch existiert). Beobachten bedeutet also das Anlegen einer Unterscheidung, aber so, daß nur eine Seite der Unterscheidung im Bezeichnen erscheint. ${ }^{3}$ Durch Beobachten gewinnen Systeme Information über ihre Umwelt. Als Elementareinheit von Information bestimmt Luhmann mit Bateson,, a difference which makes a difference" (Luhmann 1984: 68). Derjenige, für den ein Unterschied einen Unterschied macht, ist ein Beobachter. Von den Unterscheidungen, mit denen er beobachtet, hängt es ab, welche Unterschiede er wahrnimmt, d.h. was er als Information erhält. Insofern ist das, was der Beobachter als seine Umwelt sieht, sein Entwurf, seine Konstruktion.

Verstehen ist eine spezifische Weise systemischen Beobachtens. Sie setzt voraus, daß ein System in seiner Umwelt etwas als anderes System bemerkt und dessen Verhalten als Realisation systemischen Operierens interpretiert. Das verstehende System legt die Unterscheidung von System und Umwelt zugrunde. Es beobachtet, wie ein anderes System diese Unterscheidung intern für sich handhabt (vgl. Luhmann 1986: 80), und wie es seine Differenz zur Umwelt mit dem Gebrauch weiterer Unterscheidungen (vgl. Luhmann 1986: 78) in jeder Beobachtungsoperation reproduziert: als Differenz zwischen dem Bezeichneten und der dabei zugrundegelegten Unterscheidung. Verstehen eines Systems bedeutet insofern Beobachtung seines Unterscheidungsgebrauchs. Es kommt zustande,

reicht. Vgl. dazu Schneider 1991a. - Zur Integrierbarkeit von funktionaler Analyse (als der mit Systemtheorie bisher allein assoziierten Methode) und Hermeneutik vgl. Schneider 1991 und 1992.

3 In Kurzfassung dieses Sachverhalts definiert Luhmann Beobachtung als ,unterscheidende Bezeichnung“; vgl. 1990b: 52) wenn ein System unterstellt, ein anderes beobachte mit bestimmten, aus mehreren internen Möglichkeiten ausgewählten Unterscheidungen, und wenn es deshalb zu sehen glaubt, was das beobachtete System im Blick hatte. Man sieht z. B. wie jemand in einer Tasche wühlt und versteht, ,er sucht seine Zigaretten'. Suchen/haben, Zigaretten/andere Dinge, in der Tasche/woanders; dies sind die Unterscheidungen, die dabei als Unterscheidungen des verstandenen Systems angenommen werden müssen.

Ob das so erreichte Verstehen richtiges Verstehen ist, dafür gibt es keine Garantie. Das beobachtete System bleibt für den verstehenden Beobachter intransparent, bleibt eine black box. Die Bemühung um Verstehen wird dadurch nicht etwa sinnlos, sondern hat in der Kompensation dieser Intransparenz ihre Funktion. Weil Durchschauen unmöglich ist, muß verstanden werden (vgl. Luhmann 1990: 25).

Reichweite und Differenzierungsvermögen eines auf Wahrnehmung gründenden Verstehens sind begrenzt. In besonderer Weise sozial folgenreich wird Verstehen deshalb erst im Kontext von Kommunikation als dem Operationsmodus sozialer Systeme. Kommunikation begreift Luhmann als Synthese von drei Selektionen: der Selektion einer Information, der Wahl eines Verhaltens (Gesten, Wortfolgen etc.), mit dem diese Information mitgeteilt werden soll und dem Verstehen. Kommunikatives Verstehen heißt, ein Ereignis mit Hilfe der Differenz von Information und Mitteilung zu beobachten. Dabei wird im Verstehen eine Seite dieser Unterscheidung bezeichnet. Unter Normalbedingungen ist dies die Seite der Information (vgl. Luhmann 1990: 116). Angeschlossen wird in der Regel an das, was der andere gesagt hat, seltener daran, wie (mit welchen Worten, welcher Intonation, Begleitmimik etc.) er es mitteilte. Der Wechsel zur Mitteilungsseite erfolgt typisch in Situationen, in denen Verstehen problematisch erscheint, etwa wenn unklar ist, was der Unterton des Sprechers besagen soll oder warum er sich einer besonderen Formulierung bedient. Mitteilungs- und Informationsselektion treten unter solchen Umständen im Verstehen auseinander und es entsteht Bedarf für reflexive Kommunikation.

Was als Verstehen in der Kommunikation erreicht wird, entzieht sich der Steuerung jedes einzelnen Beteiligten. Es ist ein emergentes Resultat ihres Zusammenwirkens und daher nicht als Handlung zurechenbar auf Sender oder Empfänger. Zugleich aber vereinfacht sich Kommunikation ständig 
selbst zu Handlung (Luhmann 1984: 193, 212, 227ff; 1990: 38): Das, woran die Folgeäußerung des Empfängers anschließt, erscheint unter Normalbedingungen störungsfreien Ablaufs als Erzeugnis des Senders, als Resultat seines Mitteilungshandelns. Bedeutungskonstitution ist dabei das Resultat kommunikativer Attribution. Dafür ausschlaggebend ist die dritte Selektion: das Verstehen, wie es als Folge der Anschlußäußerungen des Empfängers in der Kommunikation zustande kommt. Jede Anschlußäußerung impliziert ein bestimmtes Verstehen der vorausgegangenen Äußerung, weist ihr eine bestimmte Bedeutung kommunikativ zu. Der Sprecher mag sich vielleicht mißverstanden fühlen. So z.B., wenn der Adressat die als Bitte intendierte Äußerung „Kannst du mir das Salz reichen?“ (vielleicht in scherzhafter Absicht) einfach nur mit „Ja“, d.h. als Informationsfrage, beantwortet. Belassen es beide dabei, dann ist das in der Kommunikation durch die Antwort erreichte Verstehen das Verstehen einer Informationsfrage, auch wenn beide Psychen etwas anderes verstanden haben. Und insofern gilt: Verstehen in der Kommunikation „schließt . . mehr oder weniger weitgehende Mißverständnisse als normal ein" (Luhmann 1984: 196). ${ }^{4}$ In der Kommunikation aber zählt, was durch Anschlußäußerungen zustande kommt, solange als Verstehen, wie kein Einspruch erhoben und etwas als Mißverständnis deklariert wird.

Was verstanden wird, ist weder determiniert noch limitiert durch den Informationsgehalt, um dessen Mitteilung es dem Sender ging: „... der Gebrauch von sprachlich fixiertem Sinn (gibt) der Kommunikation immense Überschüsse an semantischen Selektionsmöglichkeiten, die zu Informationen verarbeitet werden können" (Luhmann 1990: 27). Diese Überschüsse werden kommunikativ in dem Maße entbunden, wie die Selektionshorizonte zwischen mitteilendem System und verstehenden Systemen auseinandertreten, d.h. vor allem unter den Bedingungen schriftlicher Kommunikation und der Kommunikation unter Bedingungen funktionaler Differenzierung des Gesellschaftssystems.

\footnotetext{
Jedoch: „Das Verstehen und Mißverstehen einschlieBende Verstehen versteht sich immer als Verstehen (und nicht als Mißverstehen), denn als Mißverstehen würde es sein Beobachten nicht fortsetzen, sondern einstellen“ (1986: 85f). Es bedarf deshalb besonderer Einrichtungen und Kontrollmöglichkeiten, soll nicht "die Mißverstehenskomponente . . . so hoch sein, daß eine Weiterführung der Kommunikation unwahrscheinlich wird“ (1984: 217f).
}

Mit der Entstehung von Schrift und Buchdruck löst sich Kommunikation aus der Interaktion zwischen Anwesenden. Zeitlich wie räumlich weit entfernte Adressaten können nun erreicht werden, für die anderes neu oder selbstverständlich, fremd oder vertraut ist, als für den Autor der Mitteilung. Mit dem Auseinanderdriften der Selektionshorizonte divergiert der semantische Gehalt, den Autor und Leser mit einem Text verbinden können, auf nicht mehr zu übersehende und praktisch unkontrollierbare Weise. Verstehen wird deshalb im Kontext der Aneignung der schriftlichen Überlieferung zuerst als Dauerproblem sichtbar und thematisch. Mit der Expansion ihrer Reichweite wird es zugleich unwahrscheinlich, daß Kommunikation auf Aufmerksamkeit trifft und die Adressaten dazu motivieren kann, die mitgeteilten Informationen ,als Prämisse eigenen Verhaltens" zu übernehmen (Luhmann 1984: 218). Auf beide Probleme reagiert die binäre $\mathrm{Co}$ dierung von Kommunikation: Binäre Codierung heißt Etablierung spezifischer Leitdifferenzen wie wahr/falsch, recht/unrecht, schön/häßlich, glauben/nicht glauben etc., die als beobachtungsleitende Unterscheidungen die Selektion von Informationen zu Mitteilungszwecken ebenso wie die Informationsverarbeitung im Verstehen instruieren. Solche semantischen Codes sind zugleich Präferenzcodes. „Sie suggerieren, da $\beta$ es besser sei, sich für den positiven Wert als für den negativen zu entscheiden“ (Luhmann 1987: 19). Binäre Kommunikationscodes erfüllen demnach eine Doppelfunktion: Sie organisieren Kontexte der Selektion von Sinn und strukturieren so die Informationserzeugung und -verarbeitung auf grundsätzlich erwartbare Weise vor (Luhmann 1987: 15), und sie motivieren zur Annahme von Kommunikationen, die sich dem positiven Wert eines Codes zuordnen lassen.

Binär codierte Kommunikation kann bestimmte soziale Funktionen bedienen, etwa für Erwartungssicherheit in Konfliktfällen sorgen (Recht) oder Erkenntniswachstum wahrscheinlich machen (Wissenschaft). Die funktionale Autonomisierung sozialer Teilsysteme wie Recht, Wissenschaft, Wirtschaft, Politik impliziert daher zugleich die "funktionale Differenzierung der Codes“ (Luhmann 1987: 21). Durch die funktionale Differenzierung des Gesellschaftssystems wird ein Nebeneinander sozialer Großkontexte der Informationsverarbeitung erzeugt, das ein synchronisches Pendant bildet zur diachronischen Differenzierung der Selektionshorizonte, die historisch das Verstehen in Bereichen der Tradierung von vorbildlichen Tex- 
ten als Problem hervortreten ließ: ${ }^{5}$ „Unterschiedliche Codes führen zu einer unterschiedlichen Qualifizierung von Informationen, weil sie den Informationswert der Information auf unterschiedliche Selektionshorizonte beziehen" (Luhmann 1987: 21). So mag etwa die Mitteilung über Durchführung und Ergebnisse einer Tierversuchsreihe. in der ein Pharmakonzern ein neues Medikament erprobt hat, im ökonomischen System als Hinweis auf zukünftige Erträge bewertet werden und die Aktienkurse in die Höhe treiben, im Gesundheitssystem Vorbereitungen auf die Erweiterung therapeutischer Möglichkeiten veranlassen, im Wissenschaftssystem die Überprüfung von Theorien anregen, die mit diesem Ergebnis unvereinbar sind, und im Rechtssystem zu einer Ermittlung wegen Verdachts auf Verstoß gegen das Tierschutzgesetz führen. Was hier jeweils anders ausfällt, ist das, was als Verstehen - ablesbar an den unterschiedlichen Anschlüssen - erreicht wird.

Die Komplexität kommunikativer Verstehensmöglichkeiten ( $=$ Anschlußalternativen) übersteigt den Rahmen dessen, was jedes Einzelindividuum für sich als Informationssinn psychisch aktualisieren und zur Grundlage eigenen Anschlußhandelns machen kann. Möglich ist dies nur, weil über den Informationsgehalt von Kommunikation im Verstehen entschieden wird. Dem Autor einer Mitteilung ist dadurch die Kontrolle über ihre psychischen und kommunikativen Sinneffekte entzogen. Was als gemeinsamer Knotenpunkt bleibt, von dem aus die verschiedenen Sinneffekte ihren Ausgang nehmen, ist das zitierfähige Mitteilungsereignis. Variabel und zukunftsoffen sind demgegenüber die Informationsgehalte, die dieser Mitteilung im Verstehen abgewonnen werden können. Der verstandene Sinn kristallisiert zwar an der Mitteilung und ist an das Mitteilungsereignis gebunden. Das Verstehen jedoch ist produktiv. Der Informations-Mehrwert, der in der Beziehung des Verstehens auf die Mitteilung emergiert, ist nicht auf eine der beiden Seiten rückführbar. Er kann deshalb nicht als primäres Resultat intentionalen (Mitteilungs-)Handelns rekonstruiert werden, sondern muß als Ergebnis von Kommunikation als nicht weiter auflösbarer Einheit betrachtet werden (vgl. Luhmann 1984: 227).

5 Gadamer (1974: 1062ff) verfolgt die Entwicklung methodisch angeleiteter Formen des Verstehens bis in die Antike zurück, sieht aber erst seit Schleiermacher die Stufe einer allgemeinen Theorie des Verstehens erreicht.
Die systemtheoretische Beschreibung kommunikativer Sinnkonstitution läßt die hohe Unwahrscheinlichkeit des Richtig-Verstehens sichtbar werden. Als Folge soziokultureller Evolution kann zwar unter Normalbedingungen davon ausgegangen werden, daß kommunikative Anschlußselektionen „tendenziell eher im Bereich des RichtigVerstanden-Habens liegen“; jedoch ,wenn Verstehensansprüche steigen - so ... nicht zuletzt im Kontext von Theoriediskussionen über ,Verstehen" - stellt sich die normale Wahrscheinlichkeit des Mißverstehens bei größeren Schwierigkeiten kommunikativer Kontrolle wieder her" (Luhmann 1986: 87) ${ }^{6}$ Unter den Bedingungen solcher Verständigungskrisen bietet der Übergang zur Beobachtung des Beobachters (Beobachtung zweiter Ordnung) die Chance, die Wahrscheinlichkeit des Verstehens wieder zu steigern.

Begibt sich das Verstehen auf die Ebene der Beobachtung zweiter Ordnung, dann unterstellt es nicht mehr einfach bestimmte Unterscheidungen als Unterscheidungen eines anderen Systems, um nur zu fragen, was dessen Mitteilungen bedeuten. Es fragt vielmehr danach wie das mitteilende System oder andere verstehende Systeme die Welt beobachten, d.h. im Kontext welcher Unterscheidungen das Mitgeteilte für andere zur Information wird. Wer die Unterscheidungen kennt, die ein anderer in seinem Beobachten zugrunde legt, der kann versuchen, so zu sehen, wie der andere sieht, indem er die gleichen Unterscheidungen operativ gebraucht, sein Beobachten kopiert und die Beobachtungsergebnisse des anderen für sich herstellt, nachkonstruiert, nachvollzieht. Er gewinnt dadurch kein Wissen über die ,eigentliche' Bedeutung einer Mitteilung oder die ,wirkliche' Beschaffenheit der Welt, wohl aber darüber, ,wie sie intersubjektiv übereinstimmend konstruiert werden kann mit der Folge, daß die Kommunikation fortgesetzt werden kann und nicht mangels Verständigungsmöglichkeiten abgebrochen werden muß" (Luhmann 1991: 150). ${ }^{7}$

6 Luhmann (a.a.O.) fährt fort: „Zum Teil können diese Schwierigkeiten dann aber dadurch kompensiert werden, daß der ausdifferenzierte Sonderkontext auch Mißverständnisse fruchtbar werden läßt."

7 Er kann darüber hinaus die Unterscheidungen, die andere verwenden, von anderen Unterscheidungen unterscheiden, die statt dessen gebraucht werden können, und so sehen, was andere nicht sehen, so lange sie mit ihren Unterscheidungen die Welt beobachten: die Selektivität und Kontingenz ihres Beobachtens. 
Aber wieweit es dem Beobachter eines Beobachters auch gelingen mag, dessen Unterscheidungen zu erkennen, seine Beobachtungen zu reproduzieren, gleichsam mit seinen Augen (denn das sind seine Unterscheidungen) zu sehen: Die Differenz der systemischen Perspektiven bleibt dabei erhalten. Ihre totalisierende Verschmelzung erscheint ausgeschlossen. Das Verstehen eines anderen Systems geschieht im Binnenhorizont des verstehenden Systems, das seine Unterscheidungen operativ (als Beobachter erster Ordnung) verwenden muB, um sehen zu können, welche Unterscheidungen andere Systeme gebrauchen (vgl. Luhmann 1991a: 68). ${ }^{8}$ Es kann über seine Grenzen nicht hinausgreifen, am Operieren des verstandenen Systems unmittelbar partizipieren oder sich mit ihm in einem für beide externen $Z$ wischenbereich treffen. Systeme bleiben füreinander und für sich selbst grundsätzlich intransparent. Jedes Verstehen operiert mit drastischen Vereinfachungen, kann die operative Komplexität des beobachteten Systems nicht erreichen, ist nur Konstruktion des verstehenden Systems, das damit versucht, Weißheit in einem schwarzen Kasten zu erzeugen und hat genau darin seine Funktion.

\section{Verstehen in Funktionssystemen: Am Beispiel Kunst}

Die systemtheoretische Kommunikationstheorie betont die autonome Rolle des Verstehens, das sich nicht auf die Duplizierung von intendiertem Sinn festlegen läßt. Die Ausdifferenzierung sozialer Funktionssysteme diversifiziert die Möglichkeiten des Verstehens in der Gesellschaft, weil sie unterschiedliche Kontexte für die Auswahl kommunikativer Anschlüsse etabliert. Systemintern steigert sie zugleich die Möglichkeiten der Disziplinierung des Verstehens. Durch Bindung an systemeigene Voraussetzungen, an den jeweiligen binären Code und an Programme, die Kriterien für die Zuordnung von Kommunikationen zu den CodeWerten bereitstellen (vgl. Luhmann 1990: 401ff), wird festgelegt, was als adäquates Verstehen im $S y$ stem

8 So operiert das wissenschaftliche Beobachten der Systemtheorie mit Hilfe der Unterscheidung wahr/ unwahr, wenn sie andere Funktionssysteme auf deren Leitunterscheidungen hin beobachtet. Sie tut dies auch, wenn sie ihren Blick auf die Wissenschaft richtet und so als Instanz der Selbstbeobachtung des Wissenschaftssystems im Wissenschaftssystem fungiert. gilt. Das Verstehen von Kunstwerken im Kunstsystem ist ein instruktives Beispiel dafür, wie das geschieht, und welche Anforderungsniveaus dadurch erreichbar sind.

Das einzelne Kunstwerk kann verstanden werden als „Kompaktkommunikation oder auch als Programm für zahllose Kommunikationen über das Kunstwerk“ (Luhmann 1986a: 627). Kunstwerke organisieren die Beteiligung an Kommunikation und stellen sie unter spezifische Restriktionen. Sie "disziplinieren" das sich auf sie richtende Beobachten (Verstehen), ,indem sie dem Beobachter Unterscheidungen vorgeben, an die er sich zu halten hat, wenn er überhaupt an Kunst teilnehmen will" (Luhmann 1990c: 11).

Der Begriff des Beobachtens, wie Luhmann ihn hier gebraucht, übergreift das Herstellen und $\mathrm{Be}$ trachten eines Kunstwerks (vgl. Luhmann 1990c: 22). Am Prozeß der Herstellung eines Kunstwerkes ist der „Künstler . . . als Beobachter beteiligt " (1990c: 21). ${ }^{9}$ Luhmann spricht auch von binärer Codierung der Arbeit am Kunstwerk, die sich "laufend orientiert an Unterscheidungen wie stimmig/unstimmig, belebend/tötend, passend/unpassend" (1990c: 29). Ausgehend von einer ersten und insofern voraussetzungslosen Differenz, dem „ZZfall des Anfangs“ (1990c: 11), entsteht "das Werk . . . in einer Art Individualgeschichte des Einzelfalles, es kontrolliert die dazu notwendigen Entscheidungen ${ }^{10}$ und nimmt auf diese Weise den Künstler und im Nachvollzug andere Beobachter

9 Luhmann fügt, naheliegende Bedenken antizipierend, hinzu: „Daß er auch Handgriffe beisteuern muß und Handgriffe bis zu einer unbewußten Automatik des Könnens beherrschen muß, widerspricht dem nicht" (a.a.O.).

10 Die Entscheidungen betreffen den sequenziellen Einsatz der Mittel. Als Mittel der Kunst gelten ihre Unterscheidungen (Luhmann 1990c: 20). Ein Unterschied wird erst dadurch zur Information, daB er für ein beobachtendes System einen Unterschied macht, d.h. eine Frage, ein Entscheidungsproblem des Systems löst (vgl. Luhmann 1990c: 14). In Übereinstimmung damit charakterisiert Luhmann künstlerische Produktion auch als Problem/Lösungssequenz: „Man kann sich die Herstellung eines Kunstwerks als einen Entscheidungsprozeß $\mathrm{B}$ vorstellen, der Probleme sieht und unter Erwägung von Alternativen Problemlösungen sucht; oder auch etwas festlegt, um damit ein Problem zu erzeugen, das es ermöglicht, das Festgelegte als Lösung eines Problems zu behandeln" (Luhmann 1990c: 21). Von hier aus lassen sich unmittelbare Parallelen zur hermeneutischen „Logik von Frage und Antwort" (Gollingwood; Gadamer; s.u.) ziehen. 
als Beobachter erster Ordnung in Anspruch" (1990c: 29).

Weil Luhmann hier die vom Künstler im Herstellungsprozeß getroffenen Entscheidungen identifiziert mit dem Informationsgehalt des Werkes, versteht der Betrachter, der das Werk versteht, per Implikation die Unterscheidungen, von denen sich der Künstler bei der Produktion leiten ließ, mithin also auch den Künstler selbst. ${ }^{11}$ Der Betrachter braucht an den Künstler dabei nicht zu denken, sondern mag nur das Werk im Auge haben. Als Be-

11 Assoziationen zu Schleiermacher sind hier nicht ganz von der Hand zu weisen: Sie betreffen den Nachvollzug der Entscheidungen des Künstlers in der Herstellung, soweit sie ihren Niederschlag finden in den Unterscheidungen des Kunstwerks; diese Aufgabe weist Schleiermacher der „ästhetischen Kritik“ (damit also dem Verstehen von Kunst auf der Ebene der Beobachtung zweiter Ordnung) zu. Sie soll das „innere Verfahren“ des Künstlers, den „ganzen Hergang der Komposition vom ersten Entwurf bis zur letzten Ausführung" nachzeichnen (vgl. Schleiermacher 1977: 324f).

Die Form des Verstehens, von der Schleiermacher hier spricht, ist die psychologische Auslegung, die er spezifischer noch als "technische“ (vgl. 1977: 167f) bezeichnet. Ihr geht es um „vollkommenes Verstehen des Stil“" (1977: 168), um die Rekonstruktion der "Individualităt der Kombination und Darstellung“, nicht um das Verstehen des Künstlers in seiner psychischen Totalität (vgl. Schleiermacher 1977: 172). Die „beste Probe“ für das Gelingen dieser Aufgabe sei „ohnstreitig die Nachahmung“ (1977: 168). Der Erwerb der Fähigkeit zur Reproduktion, zur Herstellung einer Kopie, wird zum Gütekriterium für das erreichte Verstehen. Schleiermachers Konzept des psychologischen Verstehens weist darin zurück auf den Gedanken Vicos, nach dem wir nur das verstehen, was wir machen können (vgl. Böhler 1981: 490ff; Jauß 1981: 466), ein Gedanke, den auch Luhmann in Zusammenhang bringt mit der Beobachtung zweiter Ordnung (1991: 150, FuBn. 12).

Was bei Schleiermacher freilich fehlt, und gerade darauf kommt es hier an, ist ein Analogon zum Gedanken der Konditionierung des Verstehens durch systemspezifische Prämissen. Ganz anders als bei Luhmann, kann deshalb zwischen dem Verstehen eines Kunstwerkes und dem Verstehen der psychischen Individualität des Urhebers nur undeutlich differenziert werden. Schleiermacher versucht dies mit Hilfe der Subunterscheidung zwischen einem rein psychologischen und dem technischen Verstehen innerhalb des psychologischen Verstehens: „Der relative Gegensatz des rein Psychologischen und Technischen ist bestimmter so zu fassen, daB das erste sich mehr auf das Entstehen der Gedanken aus der Gesamtheit der Lebensmomente des Individuums bezieht, das zweite mehr ein Zurückführen ist auf ein bestimmtes obachter erster Ordnung folgt er den ,im Kunstwerk festgelegten Anweisungen" (1990c: 26) unmittelbar, d.h. ohne sie als Unterscheidungen zu thematisieren. Er sieht etwa, daß nur blau und weiß auf der Leinwand erscheinen und nimmt ,Kälte‘ als Ausdrucksqualität des Bildes wahr. Aber er muß dazu nicht die Unterscheidung zwischen ,kalten' und ,warmen' Farben beobachten und die Verwendung von blau und weiß als Lösung des Problems vorstellen, wie ,Kälte ${ }^{6}$ als Ausdrucksqualität erzeugt werden kann. Die Thematisierung der Unterscheidungen erfolgt auf der $\mathrm{Be}$ obachtungsebene zweiter Ordnung. Beobachtet wird damit zugleich, welchen Unterscheidungen andere Beobachter folgen, wenn sie das Werk als Kunstwerk beobachten. Die beobachteten Beobachter, das ist der herstellende Künstler und das sind andere Betrachter: „.. . man nimmt wahr, daß und wie einem zugemutet wird wahrzunehmen, und damit verbindet sich zwangsläufig die Vorstellung der gleichen bzw. abweichenden Wahrnehmung anderer. ... Durch die ins Werk hineinkomponierten Unterscheidungen ist ein Beobachten des Beobachtens anderer möglich, und erst auf dieser Ebene bildet sich das Kommunikationssystem Kunst" (Luhmann 1990c: 27).

Die Unterscheidungen, um die es dabei geht, sind jedoch nur solche Unterscheidungen, die auf den binären Core des Kunstsystems (schön/häßlich) beziehbar sind, und die - selbst Sedimente vergangener Kunstkommunikation - als strukturierende Prämissen weiterer Kommunikation im Kunstsystem fungieren. Luhmann spricht in diesem $\mathrm{Zu}$ sammenhang von Stil (vgl. 1986a: 632ff; 1990c: 28f). ${ }^{12}$ Die Funktion des Stils eines Kunstwerkes ist es, erkennbar zu machen, "was es anderen Kunstwerken verdankt und was es für weitere neue Kunstwerke bedeutet“, und damit „... . den Beitrag des Kunstwerkes zur Autopoiesis der Kunst zu organisieren“ (1986a: 632). Auf der Ebene des Stils ist das Repertoire der Unterscheidungen verankert, die das Beobachten von Kunst als Kunst in der Herstellung wie im Betrachten instruieren. Nicht kanonisierte Anforderungen, von de-

Denken und Darstellenwollen, woraus sich Reihen entwickeln“ (Schleiermacher 1977: 181). Noch an der Formulierung ihres Unterschieds wird deutlich, $\mathrm{da} \beta$ technische und rein psychologische Auslegung, das Verstehen der Komposition des Werks und das Verstehen der Individualität des Künstlers verschwimmend ineinander übergehen.

12 An anderer Stelle nennt Luhmann Kunstdogmatiken als Programme des Kunstsystems (vgl. Luhmann 1981: 251f und 262). 
nen nicht abgewichen werden dürfte, sind damit gemeint. Abweichungen sind im Gegenteil notwendig, um ein Werk als autonomes Einzelkunstwerk zu legitimieren. In der erkennbaren und durch die Ebene des Stils erst erkennbar gemachten Abweichung von anderen gewinnt es sein individuelles Profil, seine Einzigartigkeit. „Die Unterscheidung von Stil und Einzigartigkeit des Kunstwerks repräsentiert mithin die Differenz der Beobachtung zweiter und erster Ordnung und formuliert mit einer auf Kunst bezogenen Spezifik, daß Beobachtungen auf beiden Ebenen in einem Verhältnis der Komplementarität praktiziert werden müssen" (Luhmann 1990c: 29).

Die Beobachtung von Kunstwerken ist so zwar angelegt auf den Nachvollzug der Unterscheidungen, die den Künstler im Herstellungsprozeß leiteten. Sie richtet sich dabei aber nicht auf das Verstehen seiner persönlichen Eigenarten, nicht auf seine privaten Obsessionen, Liebhabereien oder Alltagssorgen (vgl. Luhmann 1990c: 26). Das Beobachten des Betrachters ist programmiertes Beobachten. Sofern es Beobachtung im Kunstsystem sein will, schlägt deshalb der Eigensinn der kommunikativen Anschlußbedingungen im System gegenüber den Darstellungsintentionen des Künstlers durch, so daß etwa noch ,die Leugnung jeder Ausdrucksabsicht .... als besonders raffinierte, versteckte, hintersinnige Ausdrucksabsicht aufgefaßt (wird)" (Luhmann 1986a: 623). Mit Goffman spricht Luhmann auch vom "frame effect“ des systemischen Kontextes. Der Selektionshorizont des Systems ist es, der festlegt, welche Bedeutung ein Kunstwerk in der Kommunikation gewinnen kann, unabhängig von den Intentionen seines Urhebers. Was ein Kunstwerk bedeutet, wird fixiert durch das Verstehen, wie es in der Kommunikation des Kunstsystems erreicht wird. Wie es verstanden wird, hängt $a b$ vom Selektionshorizont der Betrachter, der abhängig ist vom Selektionshorizont des Systems. Soweit auch der Künstler sich bei der Herstellung von Unterscheidungen aus dem Repertoire des Systems leiten läßt, kann er damit rechnen, daß die Betrachter das Werk entlang dieser Unterscheidungen verstehen. Operiert er dagegen aus einem abweichenden Selektionshorizont, dann wird sein Werk anders verstanden werden als es seinen Absichten entspricht. Wer abweicht, wer anderes zum Ausdruck bringen will, als im System vorgesehen, der muß scheitern. Er muß ertragen, $\mathrm{da} \beta$ er mißverstanden wird, oder das System verlassen: „.. . statt sich psychischen Individualitäten (welchen auch?) anzupassen, operiert das System mit Inklusion/Exklusion je nach dem, ob formgemäß (d.h. entsprechend den Formen = Unterscheidungen des Systems; W.L.S.) beobachtet wird oder nicht" (Luhmann 1990c: 26).

\section{Hermeneutik und Systemtheorie als Reflexionstheorien des Verstehens}

Bevor wir uns der Darstellung von Gadamers Hermeneutik zuwenden, soll noch geklärt werden, wie sich die grundlegenden Thematisierungsperspektiven zueinander verhalten, aus der die Hermeneutik und die Systemtheorie Verstehen analysieren. Was dabei zunächst auf der Hand zu liegen scheint ist, daß Verstehen für die Hermeneutik Aufgabe, für die Systemtheorie dagegen ein kontingentes Faktum innerhalb ihres Beobachtungsbereichs darstellt (vgl. entsprechend Kneer/Nassehi 1991: 354). Doch sehen wir zu, welche Ausformung diese Differenz annimmt und was daraus für die theoretische Bestimmung des Verhältnisses zwischen Hermeneutik und Systemtheorie folgt.

Verstehen kommt in die Systemtheorie, wir hatten es schon gesagt, als Antwort auf ein Bezugsproblem: das Problem der Intransparenz psychischer und sozialer Systeme füreinander. Weil dieses Bezugsproblem letztlich unlösbar ist, wirkt es als kontinuierlicher Antrieb für Strukturbildung. Verstehen erscheint damit funktional notwendig, schließt aber aus dem gleichen Grunde unaufhebbar Mißverstehen ein und nicht aus. In evolutionärer Perspektive fragt die Systemtheorie nach den empirischen Bedingungen der Möglichkeit, die das Unwahrscheinliche, daß Verstehen zustande kommt, wahrscheinlich gemacht haben. Und sie fragt nach den strukturellen Effekten von Mißverstehen. Die Unterscheidung Verstehen/Mißverstehen kann genauer als Dekomponierung eines Bezugsproblems durch eine Unterscheidung beschrieben werden. Beide Seiten der Unterscheidung sind anschlußfähig, heben die Blockadesituation der Intransparenz zwischen einander gegenüberstehenden Beobachtern auf, ermöglichen gemeinsames Weitermachen: Glaubt man, sich verstanden zu haben, kann man sich anderen Themen zuwenden, wenn nicht, können Klärungsversuche unternommen, Metakommunikation betrieben oder ein Therapeut konsultiert werden, der einem sagen soll, wie es weitergeht, nachdem man sich zumindest darüber verständigt hat, daß man sich nicht mehr versteht. So lange überhaupt irgend etwas verstanden wird, geht Strukturbildung weiter. Insofern (aber auch nur insofern) benötigt die Sy- 
stemtheorie keine Präferenz für Verstehen im Unterschied zu Mißverstehen. Sie kann diese Präferenz in ihrem Beobachtungsbereich registrieren und eine hinreichend große Wahrscheinlichkeit für deren Erfüllung als Voraussetzung dafür ansehen, $\mathrm{da} \beta$ die Bemühungen um Verstehen nicht eingestellt werden. Für Verstehen und gegen Mißverstehen zu votieren braucht sie deshalb nicht.

Anders die Hermeneutik. Ihr geht es um die Klärung dessen, was Verstehen (im Unterschied zu Mißverstehen) heißen kann, was die Bedingungen seiner Möglichkeit sind und mit welchen methodischen Richtlinien es sich so weit als möglich erreichen läßt. Ihr Verhältnis zum Verstehen ist dem der Wissenschafts- und Erkenntnistheorie zur Wahrheit analog. Wie es dort um die Klärung des Wahrheitsbegriffs, um methodologische Regeln für die Generierung wahrer bzw. die Ausscheidung unwahrer Aussagen, um Rekonstruktion von Erkenntniszuwachs mit dem Ziel geht, die Vermehrung wahren Wissens wahrscheinlich zu machen, so geht es der Hermeneutik um Verstehen. Ihr Status ist, systemtheoretisch gesprochen, der einer Reflexionstheorie des Verstehens (so wie Wissenschafts- und Erkenntnistheorie Reflexionstheorien des Wahrheitscodes sind). Und wie die Wissenschaftstheorie an den Wahrheitscode als Präferenzcode des Wissenschaftssystems gebunden ist, so die Hermeneutik an die Präferenz für Verstehen im Unterschied zu Mißverstehen. Sie läßt sich freilich keinem spezifischen Funktionssystem zuordnen und taucht dementsprechend überall dort auf, wo Verstehensprobleme virulent werden: in der Jurisprudenz, der Theologie, der Philologie, Literatur- und Kunstwissenschaft, der Geschichte etc. Weil dort erst verstanden werden muß, um bestimmte Aussagen den Code-Werten recht/unrecht, glauben/nicht-glauben, wahr/falsch, schön/ häßlich zuordnen zu können, siedelt Hermeneutik im Bereich der Methoden, die Richtlinien für die Zuordnung zu den Code-Werten bereitstellen sollen. ${ }^{13}$ Als philosophische Hermeneutik schließlich erhebt sie Universalitätsansprüche, die so weit reichen, wie das Verstehen überhaupt. Dieser Universalitätsanspruch ist jedoch nicht exklusiv, sondern durchaus mit dem Universalitätsanspruch einer Systemtheorie vereinbar, die dem Verstehen

13 Und insofern könnte sie grundsätzlich auch für die Systemtheorie von Bedeutung sein (siehe dazu unten, Abschn. 7.). Als Ansätze zu einer hier spezifisch interessierenden sozialwissenschaftlichen Hermeneutik vgl. besonders Oevermann 1979 und 1986 sowie Soeffner 1989. die Zentralposition für die Konstitution von Kommunikation als Elementarbaustein sozialer Systeme zuweist.

Die Systemtheorie analysiert die Rolle von Verstehen und Mißverstehen für den Komplexitätsaufbau von Systemen. Als Theorie, die soziale Systeme auf ihre Handhabung von Selbstreferenz hin beobachtet, versucht sie selbst, diese Systeme nach ihrer eigenen Definition dieser Beobachtungsoperation - zu verstehen. Und als Beobachter, der sein eigenes Beobachten beobachtender Systeme an dem binären Code wahr/unwahr orientiert, muß es der Systemtheorie darum gehen, richtig zu verstehen, d.h. genau diejenigen Unterscheidungen zu treffen, die soziale Systeme in ihrem eigenen Beobachten verwenden. Als Beobachter beobachtender Systeme gebraucht die $S y$ stemtheorie selbst die Unterscheidung Verstehen' Mißverstehen nach dem Muster eines Präferenzcodes. Zugleich beansprucht sie zu zeigen, daß externe Beobachter ein binär codiertes System „nicht verstehen (können), wenn sie dessen Selbstcodierung außer acht lassen" (Luhmann 1990c: 30; vgl. auch 1984: 245). Sie trifft damit Aussagen über die Bedingungen der Möglichkeit für das (richtige) Verstehen sozialer Systeme und nimmt darauf bezogen in einem exakten Sinne die Position einer Reflexionstheorie des Verstehens, einer Hermeneutik soziale Systeme ein. Sie setzt insofern die Hermeneutik auf neuem Terrain fort. ${ }^{14}$ Dabei zeigt sie, daß Verstehen von Kommunikation mit der Figur psychologischen Verstehens, dem es um den Nachvollzug subjektiv gemeinten Sinnes geht, nicht adäquat begriffen werden kann. Denn aus der Perspektive der verschiedenen Funktionssysteme gilt, daß ein gegebenes Mitteilungsereignis nur nach Maßgabe des systemspezifischen Selektionshorizontes verstanden werden kann, und daß es deshalb in jedem von ihnen erwartbar anders verstanden wird als in allen anderen.

Anders als der Autor (bzw. der ,ursprüngliche', vom Autor antizipierte Leser) zu verstehen, das kann unter den Prämissen der Hermeneutik von

14 Und sie kann insofern den „Kontingenzeinbau“ im Hinblick auf Verstehen, den Kneer und Nassehi (1991: 353 und 355) der Hermeneutik aus systemtheoretischer Perspektive empfehlen, nicht weiter treiben, als es der Hermeneutik möglich ist und - wie gleich darzulegen - von Gadamer bereits vorexerziert wurde. Damit ist jedoch nicht behauptet, daB Hermeneutik und Systemtheorie Reflexionstheorien gleichen Typs seien. Wir kommen darauf und auf die daraus folgenden Differenzen später (vgl. unter 5.) noch zurück. 
Schleiermacher bis Dilthey nur heißen, falsch zu verstehen. ${ }^{15}$,Die Hermeneutik' freilich läßt sich darauf nicht verpflichten, wie am Beispiel Gadamers überdeutlich abzulesen ist.

\section{Gadamers philosophische Hermeneutik und ihr Verhältnis zur systemtheo- retischen Konzeption von Verstehen}

Gadamer setzt sich strikt gegenüber der Reduktion von Verstehen auf psychologisches Verstehen, auf den Nachvollzug der Sinnintentionen eines Autors oder Akteurs ab. Er tut dies mit Hilfe der Unterscheidung von Sachverstehen und Meinungsverstehen (Gadamer 1965: 278f). Meinungsverstehen entspricht dem psychologischen Verstehen Schleiermachers wie auch dem historischen Verstehen, dem es um die Restitution des ursprünglichen Sinnes von Texten, Handlungen oder Artefakten aus der Perspektive der Zeitgenossen geht. Die Bedeutung des Meinungsverstehens ist für Gadamer jedoch abgeleiteter Art. Die Führungsrolle im Prozeß der Auslegung kommt dem Sachverstehen zu. Das Sachverstehen richtet sich unmittelbar auf das zu verstehende Sinngebilde selbst. Es fragt nicht danach, was der Autor dabei im Sinn hatte, welche „gedanklichen Erlebnisse“ ihn bewegten, sondern was das Sinngebilde bedeutet (Gadamer 1965: 354). Seine Bedeutung besteht für Gadamer, darin an Collingwoods Logik von Frage und Antwort anschließend, in seinem Antwortgehalt. Es zu verstehen heißt, eine Frage (einen Fragekontext, eine

15 Ablesbar ist das etwa an folgender Bemerkung Schleiermachers zu den Ursachen des Mißverstehens: „Das Mißverstehen ist entweder Folge der Übereilung oder der Befangenheit. . . . So erklärt man hinein oder heraus, was nicht im Schriftsteller (!) liegt" (Schleiermacher 1977: 93). Schleiermacher unterscheidet zwar zwischen der psychologischen Auslegung, die den Erzeugungsproze $B$ eines Textes im Denken und aus den Lebensumständen des $\mathrm{Au}$ tors nachvollziehen soll, und der grammatischen Auslegung, welche die Textbedeutung gemäß den Regeln der Sprache zu rekonstruieren hat (vgl. Schleiermacher 1977: 77ff). Beide Weisen des Verstehens müssen jedoch unter idealisierten Bedingungen im Ergebnis koinzidieren: „Vollkommen ist aber eine jede nur dann, wenn sie die andere überflüssig macht und Beitrag gibt, um sie zu konstruieren." (Schleiermacher 1977: 79). Daß ein Text noch anderes besagen könnte, als das vom Autor Gemeinte, ist damit ausgeschlossen (vgl. auch Gadamer 1965: 172ff; Schneider 1991: 29ff).
Problemsituation) zu finden, auf die es als Antwort gelesen werden kann (vgl. Gadamer 1965: 351ff). Eine Äußerung, einen Text, ein Kunstwerk als Antwort auf eine Frage, als Lösung eines Problems zu verstehen, d.h. - wenn man Batesons Definition des Informationsbegriffs zugrunde legt - seinen Informationsgehalt zu verstehen. Wie oben bereits notiert, bestimmt Bateson die Elementareinheit von Information als einen „Unterschied, der bei einem späteren Ereignis einen Unterschied ausmacht" (Bateson 1983: 488). Der Unterschied ist Unterschied nur für ein informationsverarbeitendes System, in dem er einen bestimmten $\mathrm{Zu}$ stand selegiert: Man hört, es soll kälter werden und entschließt sich, den Mantel mitzunehmen. ,Kälter werden" wird hier von ,warm bleiben' unterschieden und auf die Alternative ,Mantel zuhause lassen oder mitnehmen' bezogen. Die Bezeichnung der einen Seite der ersten Unterscheidung informiert darüber, welche Seite der zweiten Unterscheidung zu bezeichnen ist und löst damit ein Entscheidungsproblem. Die Bedeutung der Aussage "Es wird kälter" ist hier, daß sie auf die Frage antwortet, „Soll ich den Mantel mitnehmen oder nicht?". ${ }^{16}$ In der Annahme, daß Bedeutung auf die Relation von Frage und Antwort, von Problem und Problemlösung ${ }^{17}$ zurückgeführt werden kann, kommen Collingwoods Logik von Frage und Antwort (vgl. Collingwood 1955: 30ff; 1957: 21ff) und der kybernetische Informationsbegriff, wie ihn Luhmann in AnschluB an Bateson zugrunde legt, exakt zur Deckung. Aus dieser Annahme ergeben sich jedoch spezifische Folgeprobleme für das Verstehen: Wenn die Bedeutung einer Äußerung, eines Textes ${ }^{18}$ etc. relativ auf den Selektionshorizont ( $=$ die beobachtungsleitenden Unterscheidungen) des verstehenden Systems ist, wie kann dann noch angenommen werden, daß Sinn-

16 Dabei muß sich die Frage als offene Frage gar nicht gestellt haben. Sie ist u.U. nur aus der Antwort rekonstruierbar als präsupponierte Frage, die vorausgesetzt werden muß, wenn für einen Beobachter einsichtig werden soll, wie ein System von Input-Daten zu Informationen kommt.

17 Mit Luhmann (1990: 422f) betrachten wir das Schema Problem/Problemlösung als "de-sozialisierten“ Abkömmling der dialogischen Unterscheidung von Frage und Antwort.

18 Für Gadamer (wie übrigens auch für Ricoeur; vgl. 1978) hat das Verstehen von Texten paradigmatischen Charakter für Verstehen überhaupt. Deshalb und aus Gründen der Vereinfachung werden wir im folgenden meist nur von Texten sprechen. 
gebilde einen einheitlich bestimmten bzw. bestimmbaren Bedeutungsgehalt verkörpern ${ }^{19}$

Die Lösung, die Collingwood für dieses Problem vorschlägt, deckt sich mit der Antwort, die Gadamer als die Antwort der ,romantischen Hermeneutik" von Schleiermacher bis Dilthey begreift: Garant des Textsinnes ist der Selektionshorizont des Urhebers. ${ }^{20}$ Die Frage, auf die ein Text für den Autor selbst antwortet, gilt es zu rekonstruieren. Einen Text zu verstehen heißt deshalb nachvollziehen, welche Frage er für seinen Autor beantwortete, und diese Frage zu verfehlen heißt, einen Text mißzuverstehen. Damit ist eine zweite Anforderung Collingwoods jedoch nicht ohne weiteres zur Deckung zu bringen, die verlangt, nur solche Fragen für die Auslegung zuzulassen, die sich wirklich erheben, d.h. nicht auf erkennbar fehlerhaften Voraussetzungen beruhen. ${ }^{21}$ Diese Anforderung kann der Interpret nur dadurch erfüllen, daß er seinen eigenen Selektionshorizont, sein Vorverständnis als Maßstab ins Spiel bringt. Sicherheiten für eine hinreichende Kongruenz mit dem Deutungshorizont des Autors gibt es dabei nicht. In Konfliktfällen (z.B. Hexenglauben als Präsupposition der Frage, woran man eine Hexe erkenne) bleibt der Interpret ohne Direktive, die eine Auflösung erlaubt.

Gadamer votiert hier anders. Er setzt den Interpreten an die Stelle, an der Collingwood den Autor plazierte. Der Selektionshorizont des Auslegenden wird zum Kriterium der Textbedeutung. Er muß eine vom Text beantwortete Frage finden, die sich für ihn tatsächlich erhebt. ${ }^{22}$ Der Sinn des Tex-

19 Von einer analogen Problemfassung her kommt Hirsch $(1972: 21,46)$ dazu, den subjektiven Sinn eines Textes für seinen Autor als allein maßgeblich für die Interpretation zu dogmatisieren, weil er nur so glaubt, die Voraussetzungen methodologischer Objektivität des Verstehens sichern, d.h. zwischen korrekten und falschen Interpretationen überhaupt noch unterscheiden zu können. Vgl. dazu kritisch Madison 1978.

20 Oder mit Luhmann formuliert: Die Unterscheidungen des herstellenden Beobachters.

21 Welche Komplikationen sich daraus im einzelnen ergeben, kann hier nicht weiter verfolgt werden; vgl. dazu Schneider 1991: 48ff.

22 Vgl. dazu auch Popper, der in seiner, ,Theorie des objektiven Geistes" (siehe 1984: 158ff) völlig analog zu Gadamers Bestimmung des Sinnes von Texten durch ihren Antwortgehalt Theorien, Kunstwerke, Artefakte etc. als Lösungen von Problemen betrachtet und dabei strikt zwischen den tatsächlich (d.h. aus der Interpretenperspektive) gelösten Problemen und den nach Meinung des Urhebers gelösten Problemen tes ist relativ zum Vorverständnishorizont des Interpreten und damit variabel und zukunftsoffen. Er kann neue Bedeutungsschichten gewinnen und ältere abstoßen ${ }^{23}$ unabhängig davon, was der Autor mit seinem Text im Sinn hatte. Besonders deutlich wird dies im Falle der juristischen Hermeneutik, wenn es um die Anwendung gesetzlicher Regelungen auf einen neuen Fall geht: Fällt die Anzapfung fremder Leitungen zur unbefugten Entnahme von Strom unter den Diebstahlparagraphen? Dazu mußte geklärt werden, ob der im Paragraphen 242 StGB verwendete Ausdruck "Sache“ auch auf elektrische Energie angewendet werden kann, eine Frage, die sich den Autoren dieser Regelung bzw. dem Gesetzgeber, der sie beschlossen hat, so noch nicht stellen konnte. Offensichtlich wird hier die produktive Dimension der Auslegung. ${ }^{24}$ - Gadamer generalisiert dieses Moment der „Applikation“, wie es vor allem konstitutiv für die juristische und theologische Hermeneutik ist. In jedem Falle wendet der Interpret den auszulegenden Text an auf die historische Situation in der er selbst steht und aus der heraus er interpretiert. Dies darf nicht, wie oft geschehen (vgl. u.a. Betti 1962: 49ff; Apel 1971a: 31ff; abweichend Böhler 1981: 504ff) als Aufforderung zur praktischen Aktualisierung für die Gegenwart durch Ableitung normativ verbindlicher Handlungsorientierungen mißverstanden werden. Worum es Gadamer geht, ist vielmehr, daß jede Interpretation eine „situationsbe-

unterscheidet: ,Jemand, der an einem Problem arbeitet, kann selten genau angeben, was sein Problem ist (ehe er eine Lösung gefunden hat); und selbst wenn er sein Problem erklären kann, kann er sich irren. Keplers bewußtes Problem etwa war die Entdeckung der Harmonie der Welt; doch wir können sagen, das Problem, das er löste, war die mathematische Beschreibung der Bewegung in einer Menge von Zwei-Körper-Planetensystemen" (1984: 257). Zur Untersuchung der Entsprechungen zwischen Gadamers Hermeneutik und Poppers Verstehenskonzept vgl. Schneider 1991: 63-90.

$23 \mathrm{Zu}$ hier anknüpfenden Komplizierungen des Collingwood/Gadamerschen Schemas von Frage und Antwort vgl. Marquard 1987: 118; Blumenberg 1966.

24 Anders als im Bürgerlichen Recht setzt das im Strafrecht bestehende Analogieverbot der Auslegung jedoch enge Grenzen. Die Anwendbarkeit des Paragraphen 242 für solche Fälle blieb deshalb unsicher. Dies führte schließlich dazu, daß durch Gesetz vom 9.4.1900 der Paragraph 248c (Entziehung elektrischer Energie) eingeführt wurde (vgl. Schönke/ Schröder: Paragraph 248c, RN1). 
zogene Aneignung"25 einschließt. Die Situation des Interpreten aber - das ist der historisch bestimmte Horizont seines sachlichen Vorverständnisses, sein Selektionshorizont, das Repertoire seiner Unterscheidungen, das er in jeder Auslegung ins Spiel bringt und als Bedingung der Möglichkeit des Verstehens ins Spiel bringen $m u \beta$.

Die Vor-Urteile des Interpreten, die Sinn-Erwartungen, die er an einen Text heranträgt, sind daher für Gadamer nicht eine bloße Quelle der Trübung eines zu ermittelnden ursprünglichen Sinnes. Sie gelten ihm vielmehr als konstitutives Moment jeder Auslegung. Die Strukturierung des Auslegungsprozesses durch verständnisleitende Vorurteile ist unhintergehbar. Womit sonst sollte der Interpret beginnen, sofern er keine metaphysischen Fähigkeiten, telepathischen Verbindungen zum Autor, Verbalinspiration o.ä. in Anspruch nehmen kann? Erst im Fortgang der Auslegung selbst kann zwischen verstehensförderlichen und -hinderlichen Vor-Urteilen unterschieden werden durch sukzessive Selektion derjenigen Vorannahmen, die den verständlichen Gehalt des Textes maximieren. Gadamers Hermeneutik erfüllt hier durchaus die Ansprüche des Popperschen Falsifikationismus. Dadurch, daß der Ausleger seine verständnisleitenden Vormeinungen ins Spiel bringt, setzt er sie zugleich aufs Spiel. Sie können am Text scheitern und den Interpreten dann veranlassen, diese Annahmen zu prüfen. Von zentraler Bedeutung dafür ist das sogenannte „Vorurteil der Vollkommenheit" (Gadamer 1965: 278), das Gadamer als Einstellung des Interpreten gegenüber dem Text verlangt. Nur dann, wenn er damit rechnet, daß der Text, wenn er seinen Vorannahmen widerspricht, gegen ihn im Recht sein könnte, kann der Interpret aus der Beschäftigung mit Texten für ihn Neues lernen. Beim „Vorurteil der Vollkommenheit" handelt es sich also um eine methodologische Maxime, deren Funktion es ist, die sachliche Informationsausbeute, die der Interpret aus einem Text gewinnen kann, zu maximieren (vgl. Gadamer 1965: 352). Deshalb - und nicht etwa als Folge einer Dogmatisierung der Autorität der Überlieferung - ist die vorschnelle Unterstellung irrationaler Prämissen auf der Seite des Textautors, die es mit aufklärerischen, ideologiekritischen, psychoanaly-

25 Vgl. Böhler 1981: 506: „Insofern die Übersetznng eines Textes über die historische Differenz eine notwendigerweise aktualisierende Erschließung ist, enthält sie eine situationsbezogene Aneignung, die jedoch keine direkte Anwendung eines, vorweg als verbindlich gesetzten, handlungsleitenden Sinns ist. “ tischen oder anderen Mitteln der Latenzbeobachtung zu durchleuchten gälte, kontraindiziert. Erst dann, wenn alle Bemühungen des Interpreten, den Text als gültige Antwort auf eine sich für ihn erhebende Frage auszulegen scheitern, erscheint es angemessen, Hypothesen über zeittypische oder für den Autor charakteristische Hintergrundannahmen in der Auslegung heranzuziehen, die dem Interpreten als irrational erscheinen. Dann erst wird es notwendig, in den Bezirk des nur noch historischen und/oder psychologischen Verstehens einzutreten, den Gadamer unter der Bezeichnung des Meinungsverstehens zusammenfaßt. So lange dieser Punkt jedoch nicht erreicht ist, darf die Ebene des Sachverstehens nicht verlassen werden, soll der Sinngehalt des Textes für den Interpreten soweit als möglich ausgelotet werden. - Gemessen an diesem Ziel ist die ,psychologische Rekonstruktion der Gedanken eines Autors eine ganz andere Frage“ (Gadamer 1965: 354). Darin kommt Gadamer mit Luhmann überein, der das Verstehen von Kommunikation strikt vom Verstehen der Person unterscheidet, die als Urheber einer Mitteilung an der Kommunikation beteiligt ist (vgl. Gadamer 1965: 354; Luhmann 1986: 96).

Diese Übereinstimmung ist keineswegs zufällig. Wie sich für Luhmann Kommunikation vom Verstehen her organisiert (vgl. 1986: 95), so wird der Sinn eines Textes für Gadamer durch dessen Auslegung, Auslegung aber als Vollzugsweise des Verstehens bestimmt (Gadamer 1965: 366). Wie Luhmann votiert Gadamer damit gegen eine handlungstheoretische Auffassung von Kommunikation, die den Sinn von Äußerungen und Texten ausschließlich durch die Sinnintentionen ihrer Urheber bestimmt sieht und dementsprechend hermeneutisches Verstehen mit psychologischem Verstehen vollkommen identifiziert. ${ }^{26}$ Hörer und Interpret gelten nicht mehr als passive Instanzen, gleichsam als siamesische Zwillinge des Autors einer Mitteilung, dazu verurteilt, dessen subjektiven Sinn zu duplizieren, sofern sie deren Sinn verstehen wollen. Die Differenz der Selektionshorizonte wird ernst genommen. Deshalb gilt grundsätzlich, ,immer übertrifft der Sinn eines Textes seinen Autor. Daher ist das Verstehen kein nur reprodukti-

26 Deutlich wird die Bindung des Textsinnes an den subjektiven Sinn des Interpreten auch in der handlungstheoretisch verkürzten Hermeneutikrezeption von Habermas; vgl. dazu Habermas 1970: 287ff; 1971: 132; 1981, Bd. I: 191 sowie die Entgegnung Gadamers auf Habermas 1970, abgedruckt in Gadamer 1986: 241ff. Zur Kritik der Habermasschen Hermeneutikrezeption siehe auch Schneider 1991: $19 \mathrm{ff}$. 
ves, sondern stets auch ein produktives Verhalten“, und insofern gilt, „daß man anders versteht, wenn man überhaupt versteht" (vgl. Gadamer 1965: 280; Hervorhebung von mir). Kommunikativer Sinn erscheint damit als emergentes Resultat doppelt kontingenter Konstitution: Der Autor fixiert die Folge der Zeichen, die Mitteilungsgestalt und etabliert damit scharfe Restriktionen für das, was als Information auf den Text zurückgeführt werden kann. Jede Deutung untersteht dem Gebot der Deckung durch den Text. Dennoch reicht die Mitteilungsgestalt nicht aus, um den Textsinn zu determinieren. Der Text bedarf des Auslegers, und er gewinnt seinen Sinn erst relativ zum jeweiligen Sinnhorizont, auf den er im Verstehen bezogen wird. Jedes Verstehen ist daher Interpretation. Interpretation aber ist nicht etwas dem Text äußerliches, kontingent hinzutretendes, denn erst im Verstehen wird er vollendet, kommt er neu zur Sprache, erhält er seinen Sinn (Gadamer 1965: $156,280,437 f, 448$ ).

Daß Gadamer dem Verstehen eine derartig zentrale Rolle für die Konstitution des Textsinnes zuweist, hat weitere Konsequenzen, die dicht an Luhmanns Konzeption von Verstehen und Kommunikation heranführen:

Es kann für Gadamer „keine richtige Auslegung ,an sich" geben“, denn ,jede Auslegung hat sich in die hermeneutische Situation zu fügen, der sie zugehört“ (1965: 375). „Jede Aneignung der Überlieferung ist eine geschichtlich andere - was nicht heißt, daß eine jede nur eine getrübte Erfassung derselben wäre: eine jede ist vielmehr die Erfahrung einer ,Ansicht' der Sache selbst“ (1965: 448). Statuiert ist damit die objektive Realität unterschiedlicher Beobachtungsperspektiven für das Verstehen. Der Sinn eines Textes kann damit auch nicht gebunden werden an die notwendig selektive Auslegung eines einzelnen Interpreten. Wie die Sinnintention des Autors, so übersteigt er notwendig ebenso die Verstehenskapazität jedes einzelnen Interpreten und ist somit nicht mehr auf eine individuelle psychische Instanz beziehbar. Virulent wird deshalb die Frage nach der Ebene, von der her der Vorgang multipler Sinnkonstitution als Einheit gedacht werden kann.

Im Hegelschen Kontext ist die Funktionsstelle einer transindividuellen Einheit durch den Begriff Geist besetzt, wird also nach dem Muster eines Makrosubjektes gedacht. Luhmann votiert für Kommunikation als Einheit, die in sich differentiell (als Einheit von drei Selektionen) konstituiert ist und nicht mehr auf ein Subjekt (da sie minde- stens zwei Prozessoren voraussetzt) zurückgeführt werden kann. Gadamer besetzt diese Stelle mit dem Begriff der Wirkungsgeschichte. Wirkungsgeschichte meint die sinnproduktive Wirkung der Geschichte, die über den Wandel der verständnisleitenden Vorurteile neue Möglichkeiten der Deutung erschließt und damit neue Sinnbezüge eines Textes freilegt (vgl. 1965: 284ff). Um die Sinnfülle eines Textes zugänglich zu machen, ist die Verschiebung des Sinnhorizontes, in dem er interpretiert wird, konstitutive Voraussetzung. Darin und nicht im Absterben von Interessenbindungen, die den Blick der Zeitgenossen trüben könnten, sieht Gadamer die produktive Wirkung des Zeitenabstandes (1965: 282).

Von hier aus läßt sich eine Parallele ziehen zum Modus der kommunikativen Reproduktion systemischer Strukturen. Wo Luhmann von Kommunikation spricht, da spricht Gadamer von Wirkungsgeschichte und Tradition, die sich als Überlieferungsgeschehen vollzieht. Die „Seinsart der Überlieferung . . . ist Sprache“. Die „sprachliche Kommunikation zwischen Gegenwart und Überlieferung" ist das Geschehen, in dem sich die Überlieferung erhält und fortbildet. Von diesem Geschehen sagt Gadamer, daß es ,nicht unser Tun an der Sache, sondern das Tun der Sache selbst ist“ (1965: 439). Das Überlieferungsgeschehen selbst wird hier zum Subjekt. ${ }^{27}$ Der Autor und seine Interpreten sind Prozessoren in diesem Geschehen.

Die Wirkungsgeschichte eines Textes kann gelesen werden als Reproduktionssequenz einer Tradition: Diese setzt sich so lange fort, wie der Text als gültige Antwort auf (alte und/oder neue) Fragen ver-

27 Die Struktur des Spiels, in dem ,das Verhalten der Spielenden nicht als ein Verhalten der Subjektivität verstanden werden dürfe, da vielmehr das Spiel es ist, das spielt, indem es die Spieler in sich einbezieht und so selber das eigentliche subjectum der Spielbewegung wird" (Gadamer 1965: 464), ist das Paradigma für die Vollzugsform des Überlieferungsgeschehens.

Kritisch dazu Frank (1985: 20ff), der Gadamer vorhält: ,Tatsächlich fällt damit dem Subjekt-als-Wirkungsgeschichte $\mathrm{zu}$, was dem Subjekt-als-tätig-sichentwerfendem-Individuum aberkannt wurde: die (durch Tradition motivierte, aber nicht unfreie) Konstitution von Sinn als aktive Verinnerung und Überschreitung des geschichtlich vorgegebenen Diskurses" (1985: 23f). 
standen werden kann. ${ }^{28}$ Reproduktion einer Tradition meint hier also nicht geschichtsenthobene Konstanz, sondern die Anschließbarkeit von Transformationen im Bestand der deutungsleitenden Vorannahmen an ein Gemeinsames, das durch die anhaltende Geltung überlieferter Texte gestiftet wird. Die kontinuierliche Geltung der biblischen Offenbarung als Grundlage der christlichen Tradition oder die Rezeption des römischen Rechts geben Beispiele für diesen Reproduktionsmodus von Traditionen als Bedeutungszusammenhängen, welche in ständiger Transformation begriffen sind und für die identische Reproduktion nur noch als imaginärer Grenzfall ohne empirisches Korrelat in Betracht kommt. Instanzen der „Applikation" als Anwendung von Texten auf immer neue Fragekontexte, wie sie im Bereich juristischer wie theologischer Auslegung selbstverständlich aufgegeben erscheint, markieren hier gleich sam die Elementarereignisse oder basalen Operationen, auf denen die Reproduktion von Traditionen gründet. „Applikation“, das ist hier zu wiederholen, meint nicht die nachträgliche praktische Anwendung eines schon verstandenen, sondern die Aneignung eines Textes aus der je spezifischen (Deutungs)Situation des Interpreten. Die Applikation als basale Einheit der Reproduktion einer Tradition kombiniert das Verstehen im Sinne Luhmanns als dritter Selektion, die kommunikativ durch mindestens eine Anschlußäußerung realisiert werden muß, mit der Annahme des verstandenen Sinnes. Die Anschlußäußerung, das ist bei Gadamer zunächst die Interpretation. Wie bei Luhmann die minimale Einheit von Kommunikation aus zwei Äußerungen besteht, von denen die zweite der ersten eine bestimmte Bedeutung kommunikativ zuweist, so ist für Gadamer die Interpretation konstitutiver Bestandteil des Textes oder Kunstwerkes selbst, denn nur als Interpretiertes gewinnt es einen bestimmten Sinn.

Dort, wo Gadamer die „Seinsweise des Kunstwerks" am Leitfaden des Spieles expliziert (1965: 97ff), tritt die konstitutive Rolle der Interpretation zuerst hervor. Gadamer reklamiert zunächst den „Primat des Spieles gegenüber dem Bewußtsein der Spielenden“ (1965: 100). „Das eigentliche Subjekt des Spieles . . . ist nicht der Spieler, sondern das Spiel selbst. Das Spiel ist es, was den

28 Und in diesem Sinne spricht Gadamer von „Horizontverschmelzung“: „Im Walten der Tradition findet ständig solche Verschmelzung statt. Denn dort wächst Altes und Neues immer wieder zu lebendiger Geltung zusammen“ (1965: 289).
Spieler im Banne hält, was ihn ins Spiel verstrickt, im Spiel hält" (1965: 102). Man ist versucht, für „Spiel“" „Kommunikation" einzusetzen, um so bei Luhmanns Diktum anzulangen, nach dem nur die „Kommunikation kommuniziert" (vgl. u.a. 1988: $884) .{ }^{29}$ Und tatsächlich sagt Gadamer, daß ,vielmehr das Spiel es ist, das spielt" (1965: 464). Das Spiel ist bestimmt durch seine „Regeln und Ordnungen“ (1965: 102) und in seinem Sein zugleich abhängig davon, daß es gespielt wird. Dies gilt auch für das Schauspiel als Kunstwerk. Die Abhängigkeit davon, daß es gespielt wird, hat zur Folge, daß die Kontingenzen seiner Darstellung ihm nicht äußerlich bleiben, sondern es mit konstituieren. „Das Kunstwerk ist nicht von der ,Kontingenz' der Zugangsbedingungen, unter denen es sich zeigt, schlechthin isolierbar . . . Es selbst gehört in die Welt hinein, der es sich darstellt. Die These ist also, daß das Sein der Kunst nicht als Gegenstand eines ästhetischen Bewußtseins bestimmt werden kann, weil umgekehrt das ästhetische Verhalten mehr ist, als es von sich weiß. Es ist Teil des Seinsvorganges der Darstellung und gehört dem Spiel als Spiel wesenhaft zu“ (1965: 111). Zur Kontingenz der Entstehung eines Kunstwerkes tritt hier die Kontingenz der Darstellungsbedingungen als zweites notwendiges Moment für die Existenz des Kunstwerkes hinzu. Der Sinn des Kunstwerkes ist notwendig doppelt kontingent bestimmt und daher weder auf seine Existenz als Tatsache im Bewußtsein seines Urhebers noch als Gegenstand ästhetischer Erfahrung, wie er sich dem Bewußtsein des Betrachters darstellt, reduzierbar. Das Spiel als Gebilde, als „ein bedeutungshaftes Ganzes, das als dieses wiederholt dargestellt und in seinem Sinn verstanden werden kann", und seine Abhängigkeit vom „Gespieltwerden“, von sei-

29 Das hermeneutische Prallelkonzept zum systemtheoretischen Kommunikationsbegriff ist bei Gadamer die Wirkungsgeschichte. Die damit berührte Frage, inwiefern Gadamer die Wirkungsgeschichte gegenüber den verstehenden Subjekten auf ähnlich weitgehende Weise autonomisiert, wie Luhmann die Kommunikation gegenüber den daran Beteiligten, kann hier nicht weiter verfolgt werden. $\mathrm{DaB}$ verschiedene Äußerungen Gadamers so gelesen werden können, belegen die obigen Zitate ebenso wie Franks Kritik am "Subjekt-als-Wirkungsgeschichte“ (vgl. Anmerk. 27). Gadamers Position hierzu erscheint jedoch nicht so eindeutig, daß ein abschlieBendes Urteil ohne eingehende interpretarorische Bemühungen möglich wäre. 
ner Darstellung, gehören zusammen (1965: 111)..$^{30}$ Der „Abstraktion der ästhetischen Unterscheidung" zwischen Werk und Darstellung, dem ,eigentlichen Konstitutivum des ästhetischen $\mathrm{Be}$ wußtseins“, setzt Gadamer „die ,ästhetische Nichtunterscheidung" entgegen" (1965: 111). Diese Nichtunterscheidung wird näher bestimmt als die Einheit „der doppelten Unterscheidung von Dichtung und ihrem Stoff und von Dichtung und Aufführung" (1965: 112) ${ }^{31}$ Der Stoff, das Thema, die zugrunde liegende Fabel zum einen, seine dichterische Gestaltung zum zweiten und schließlich die darstellerisch realisierte Interpretation kommen je in einem Sinnganzen zur Einheit. Diese Einheit entspricht im Grundsatz der Einheit der drei Selektionen Information, Mitteilung und Verstehen, die für Luhmann Kommunikation konstituiert. Der Sache nach erweist sich die Struktur, die Gadamer paradigmatisch am „Spiel der Kunst“ entfaltet und schließlich als universelle Struktur des Überlieferungsgeschehens im "Spiel der Sprache" (1965: 464f) bestimmt, als Analogon zur Struktur des Luhmannschen Kommunikationsbegriffs. Grundlage dieser Übereinstimmung ist, daß Gadamer wie Luhmann den Sinn von Texten bzw. Kommunikation nicht handlungstheoretisch, sondern aus der Perspektive des Verstehens konzipieren. Von dort kommt Gadamer zu der Annahme der grundsätzlichen Ungleichmächtigkeit zwischen dem Sinn eines Textes und seinem ,subjektiven" Sinn für den Autor sowie dessen Zeitgenossen.

30 Die unterschiedlichen Weisen der Realisation widersprechen dem nicht: Bei der ,Varietät der Ausführungen eines solchen Gebildes" handelt es sich "nicht um bloße subjektive Varietät von Auffassungen, sondern um eigene Seinsmöglichkeiten des Werks, das sich gleichsam in der Varietät seiner Aspekte selber auslegt" (1965: 112).

31 Aus dieser Einheit, welche die „eigentliche Erfahrung einer Dichtung" ausmacht, tritt heraus, wer "die zugrundeliegende Fabel auf ihre Herkunft hin betrachtet" oder "über die Auffassung, die einer Aufführung zugrunde liegt“" reflektiert, d.h. die „ästhetische Unterscheidung des Werkes von seiner Darstellung" in Anschlag bringt (1965: 112). Nicht die Legitimität einer solchen ästhetischen Reflexion möchte Gadamer bestreiten, sondern nur deren abgeleiteten und auf Vermittlung des Unterschiedenen zur Einheit hin gerichteten Status nachweisen, denn: „In Wahrheit unterstellen sie sich alle dem kritischen Leitmaßstab der ,richtigen' Darstellung (1965: 113). Grundlage ästhetischer Kritik ist die „Störung des einheitlichen Verstehens“ (1965: 113). Sie fungiert als Instanz der Meta-Kommunikation, die aufgerufen ist, wenn das Verstehen sich mit der Möglichkeit des Nicht- oder Mißverstehens konfrontiert sieht.
Psychologisches und historisches Verstehen greifen deshalb für Gadamer grundsätzlich zu kurz, wenn es um das Verstehen des Textsinnes geht.

Diese Verstehensformen sind gleichwohl für $\mathrm{Ga}$ damer nicht bedeutungslos. Das dadurch zu gewinnende Bewußtsein der Andersheit des Gegenwartshorizontes gegenüber dem Horizont der Überlieferung, aus dem der Text spricht, wird dem Interpreten als "historisches Bewußtsein“ ausdrücklich abverlangt. ${ }^{32}$ Im gelungenen Verstehen jedoch ist dieses Bewußtsein nur ,wie eine Überlagerung über einer fortwirkenden Tradition, und daher nimmt es das voneinander Abgehobene sogleich wieder zusammen, um in der Einheit des geschichtlichen Horizontes, den es sich so erwirbt, sich mit sich selbst zu vermitteln“ (1965: 290). Das wirkungsgeschichtliche Bewußtsein weiß sich so als Einheit der Differenz der verschiedenen Vorverständnishorizonte und der korrespondierenden Auslegungsmöglichkeiten eines Textes. Diese Einheit konstituiert sich mit jeder Anwendung des Textes auf die Situation des Interpreten neu als Fortsetzung des Überlieferungsgeschehens, als Erneuerung der Tradition in der Gegenwart des Interpreten.

\section{Grenzen der Kompatibilität}

Zur Einheit kommen die verschiedenen Ansichten eines Textes insofern, wie sie $i h m$ zurechenbar bleiben. Der vorverständnisrelativ variierende Sinn eines Textes wird im Überlieferungsgeschehen zur temporalen Einheit einer Transformationssequenz zusammengefaßt, die unter der Bedingung der Fortgeltung des Textes steht. Diese Einheit zerbricht mit dem Abbruch einer Tradition.

Eine solche Gesellschaft übergreifende Einheit kennt die Systemtheorie unter den Bedingungen funktionaler Differenzierung nicht. Hier treten die Funktionssysteme als unterschiedliche Großkontexte der Informationsverarbeitung mit je eigenem

32 Insofern das wirkungsgeschichtliche Bewußtsein die Andersheit des Horizonts der Überlieferung zusammen mit dem Gegenwartshorizont umgreift, ist es dem Autor und den zeitgenössischen Lesern voraus. Es versteht den Text nicht nur, wie es selbst ihn (als Beobachter erster Ordnung) versteht, sondern kann darüber hinaus verstehen, wie andere ihn vor ihm verstanden haben. Der im Bewußtsein der Wirkungsgeschichte auslegende Interpret operiert mit dem Vorteil des Beobachters zweiter Ordnung, der sehen kann, mit welchen Unterscheidungen (d.h. aus welchem Vorverständnishorizont) frühere Beobachter erster Ordnung den Text beobachtet haben. 
Selektionshorizont auseinander, ohne daß Tradition (oder eine systemübergreifende prozeduralisierte Vernunft wie bei Habermas und Apel) eine Ebene der Konstitution von Einheit als Geltungskonsens bereitstellen würden. Verstehen ist unter Bedingungen binär codierter Kommunikation grundsätzlich an den je besonderen Selektionshorizont des verstehenden Systems gebunden, das andere Systeme deshalb nur noch nach dem $\mathrm{Mu}$ ster psychologischen bzw. historischen Verstehens beobachten kann. Die Beobachtung des Beobachters hat hier zu kompensieren, daß ein in der Sache koinzidierendes Verstehen nicht mehr möglich ist. Verstanden (und damit erwartbar gemacht) werden kann nur noch, wie - d.h. aufgrund welcher Unterscheidungen - andere Systeme dazu kommen, so anders zu verstehen, wie sie verstehen. Jedes System kann jedes andere beobachten. Keines ist im Vor- oder Nachteil. Kongruenz der Beobachtungsperspektiven über die Systemgrenzen hinweg ist bei Strafe des Verlustes systemischer Identität ausgeschlossen. Was dann allenfalls noch möglich ist, ist „Kompossibilität"“33 als Kompensation der unerreichbar gewordenen Einheit der Gesellschaft.

Die Direktiven, die Luhmanns Systemtheorie einerseits, die Hermeneutik Gadamers andererseits für die Verstehenden bereithalten, divergieren dementsprechend deutlich: Offenheit für die Anrede der Überlieferung mit Aussicht auf Traditionsfortsetzung steht hier der Aufforderung gegenüber, sich auf multiperspektivisch gebrochene, heterarche Beobachtungsverhältnisse einzurichten durch Ausbildung einer moraldistanzierten Kultur der Beobachtung anderer Beobachter (vgl. Fuchs 1992: Kap. VIII.1 und VIII.2), die von vornherein nicht mehr anstrebt als nur gelegentliche und äuBerst selektive Akkordierung der Beobachtungsperspektiven, im übrigen aber gerade (und gerade dadurch) „Inkongruenz" pflegt (vgl. Luhmann/ Fuchs 1989: 223f).

Was demgegenüber bei Gadamer auffällt, ist die Marginalisierung von Traditionsbrüchen. Traditionsbrüche sind gegeben, wenn einst als verbindlich geltende Texte vom Interpreten nicht mehr als

33 „Kompossibilität“ meint Verträglichkeit der zwischen den Funktionssystemen ausgetauschten Leistungen mit den Abnahmemöglichkeiten der nachfragenden Systeme. Zur systemtheoretischen Entfaltung dieses Konzepts vgl. Fuchs 1992: 89ff. Zur Bedeutung der wechselseitigen Leistungsabhängigkeit der Funktionssysteme als Substitut für die bei Parsons unterstellte normative Einheit der Gesellschaft vgl. auch Schneider 1991: $192 f$.
Antwort auf Fragen verstanden werden können, die sich auch für ihn erheben. Die Selektionshorizonte zwischen Gegenwart und Überlieferung treten unversöhnlich auseinander. Das Sachverstehen stößt an die Grenzen seiner Möglichkeiten. Wir haben es dann mit der diachronen Parallelsituation zum Luhmannschen Szenario funktional differenzierter Systemkontexte der Informationsverarbeitung zu tun. ${ }^{34}$ Die Häufigkeit von Traditionsbrüchen ist nun einerseits eine empirische Frage. Andererseits aber muß sie sich in jedem Einzelfall erst zeigen am Scheitern des Versuchs, einen auszulegenden Text als Antwort für den Interpreten zu verstehen, sollen alle Möglichkeiten des Verstehens ausgeschöpft werden. Die für die Hermeneutik konstitutive Präferenz für Verstehen und die ihr aufgetragene Aufgabe seiner Optimierung ist es, die hier aus systematischen Gründen zu einer methodologisch begründeten Marginalisierung von Traditionsbrüchen drängt, selbst wenn empirisch anderes erwartbar erscheint. Per Implikation wird damit die Hermeneutik auch zum Treuhänder für die Fortsetzung des Überlieferungsgeschehens. Und mit Habermas (1975: 241f) kann man dies dann als das praktische Erkenntnisinteresse begreifen, das quasi-transzendental in der Struktur hermeneutischer Auslegung verankert ist.

Auch in der synchronen Dimension rechnet Gadamer durchaus mit dem Aufeinandertreffen divergenter Vorverständnishorizonte und daraus folgenden Verständigungsproblemen. Die Aufgabe der Hermeneutik ist es, so weit als möglich die Überwindung solcher Barrieren anzustreben: „Wenn man der Hermeneutik folgt, zielt vielmehr jede Anstrengung des Begreifens auf den möglichen Konsens, das mögliche Einverständnis . . ." (Gadamer 1986: 114f). Dabei fügt Gadamer jedoch ergänzend hinzu, diese Anstrengung des Begreifens müsse „.. . .selbst schon auf einem verbindenden Einverständnis beruhen, wenn je herauskommen soll, daß man sich versteht. Das ist durchaus keine dogmatische Annahme, sondern eine einfache phänomenologische Beschreibung. Wo nichts verbindet, kann auch kein Gespräch gelingen" (a.a.O.: 117, Fortsetzung des vorangegangenen Zitats). Daß das Gespräch scheitern kann,

34 Einschlägig ist vor allem das Analogon zur schwächeren Variante des Traditionsbruchs: Es können die gleichen Texte, die gleichen Mitteilungen, je nach Bezugssystem, als Antworten auf unterschiedliche Fragen gelesen, mit divergierenden Leitunterscheidungen auf Informationsgehalte abgetastet werden. 
weil die notwendige Vorverständigung, ein gemeinsamer Deutungshorizont fehlt, wird hier als Möglichkeit durchaus eingeräumt. Die Hermeneutik Gadamers scheint insoweit nicht gebunden an die Wahrheit einer empirischen Hypothese über die mögliche Realisierung von Konsens. Sie klärt die Bedingungen der Möglichkeit von Einverständnissen, die erfüllt sein müssen, sofern es zu einem Einverständnis kommt bzw. kommen soll. Ihr Geltungsanspruch als Reflexionstheorie des Verstehens ist transzendentallogischer Art. Im Blick auf ihre als universal postulierte Reichweite adoptiert sie dennoch die erwähnte Hypothese. Soll - wie Gadamer dann doch annimmt - die Herstellung von Einverständnis grundsätzlich möglich sein, müssen freilich die Prinzipien der philosophischen Hermeneutik über die Grenzen der Wissenschaft hinaus als Prinzipien praktischer Vernunft im alltäglichen Verstehen sozial verankert sein (vgl. Gadamer 1986: 117, 499f). Unter diesem Gesichtspunkt erscheint bei Gadamer der Bezug jedes Verstehens auf Wahrheit (im Sinne theoretischer Wahrheit bzw. normativ-praktischer und ästhetischer Geltungsansprüche) konstitutiv vorausgesetzt.

Aus Luhmanns Perspektive ist diese Voraussetzung allenfalls in den Funktionssystemen Wissenschaft, Recht und Kunst annähernd erfüllt. In anderen Funktionssystemen jedoch steuern andere Codes das Verstehen in der Kommunikation. An dieser Stelle baut die Systemtheorie mehr Kontingenz in das Verstehenskonzept ein, als Gadamers philosophische Hermeneutik ohne Einschränkung ihres Universalitätsanspruchs verkraften kann. Hier präsentiert sich die Systemtheorie in einem genauen Sinne als eine Hermeneutik sozialer Funktionssysteme eigenen Zuschnitts: Sie reflektiert die Bedingungen der Möglichkeit (und die Grenzen) des Verstehens nicht in einem transzendentallogischen Sinn, sondern als Theorie mit empirischem Anspruch und im Blick auf die historisch kontingente Realisierung eines spezifischen Typs sozialer Differenzierung.

Dem kann (und muß) die Hermeneutik auf der Ebene transzendentaler Reflexion nichts entgegensetzen. Was sie statt dessen versuchen kann, ist, mit ihren methodischen Mitteln zu demonstrieren, daß die Orientierung an vernünftigen Geltungsansprüchen in der Kommunikation weiter reicht, als die Systemtheorie glaubt. Wenn hermeneutisches Verstehen dabei auf systemische Grenzen möglichen Einverständnisses treffen sollte, wäre die philosophische Hermeneutik freilich nicht ,widerlegt", sondern nur angezeigt, daß sie ihren Anspruch auf universale Reichweite ein- schränken muß. Auch dann aber kann noch hermeneutisch verstanden werden, welche Differenzen zwischen Deutungshorizonten es verhindern, daß Einverständnis als Geltungskonsens zu erreichen ist.

\section{Zur Funktion von Tradition}

Die Systemtheorie gibt theoretische Gründe dafür an, warum unter den Bedingungen der modernen Gesellschaft die Erfahrung traditionsbruchanaloger Diskontinuitäten und Verständigungsgrenzen zur sozialen Normalität wird. Auch wenn dies die Reichweite ihres Anspruchs tangiert, soweit er die Ermöglichung von Einverständnis betrifft, muß sich die Hermeneutik gegenüber solchen Überlegungen u.E. nicht taub stellen. Ein Anzeichen für ihre Triftigkeit ist nicht zuletzt die Differenzierung der Hermeneutik selbst entlang der Grenzen unterschiedlicher systemischer Anwendungskontexte (insbesondere Recht, Religion, Kunst und parallel dazu die wissenschaftlichen Disziplinen Jurisprudenz, Theologie, Kunst- und Literaturwissenschaft) mit divergierenden Anforderungen der „Applikation“ (vgl. dazu u.a. Herzog 1981 und 1981a; Böhler 1981: 504ff). Die Differenzierung funktionsspezifischer Selektionskontexte läßt unter systemtheoretischen Prämissen den Gedanken eines einheitlichen Überlieferungsgeschehens nicht mehr zu. Was möglich bleibt, ist Traditionsbildung auf der Ebene sozialer Teilsysteme. Ein solchermaßen pluralisiertes Überlieferungsgeschehen könnte aus systemtheoretischer Perspektive, so unsere Vermutung, einen Platz im Kontext intrasystemischer Identitätsreflexion finden. Prüfen wir diese Vermutung knapp am Beispiel des Wissenschaftssystems.

Durch Reflexion konditioniert ein System das eigene Operieren, indem es sich an seiner Differenz zur Umwelt orientiert. Dazu muß es seine eigene Einheit thematisieren und Selbstbeschreibungen anfertigen, an die es sich in der weiteren Kommunikation halten kann (vgl. Luhmann 1984: 617f). Reflexion auf der Ebene des Wissenschaftssystems bedeutet Reflexion des Codes. Dieser Aufgabe widmen sich Erkenntnistheorie und Wissenschaftstheorie. Die Grenzziehung zwischen wissenschaftlichen Disziplinen und die darauf bezogene Reflexion ist demgegenüber vor allem auf der Ebene der Programme (Theorien und Methoden; vgl. Luhmann 1990: 401ff) und konstituierender Problemstellungen (vgl. Luhmann 1981b) möglich. Spezifiziert auf diese Ebene der disziplinären Dif- 
ferenzierung formuliert Luhmann entsprechend: „Die Reflexion betrifft die jeweiligen Problemund Theorietraditionen, an denen man Thematiken als ,wissenschaftlich" erkennt" (Luhmann 1981a: 324). Für Forschungen, die als „Grundlagenforschung" auf der Ebene disziplinärer Reflexion operieren und ,sich um Steigerung des Auflösevermögens oder des Konsistenzniveaus der Begriffe und Theorien bemühen" gilt: „Sie haben immer verstärkt historische Implikationen. Ohne hier Anschluß zu suchen, wäre wissenschaftliches Arbeiten nicht möglich, weil als solches nicht identifizierbar. Das schließt nicht aus, jede bestimmte Problem- oder Theorietradition (mit Hilfe anderer!) in Frage zu stellen. Anschlußzwang ist mithin nicht nur eine Frage der Ökonomie und des Vermeidens unnötiger Doppelarbeit: es ist eine Frage der Identität" (Luhmann 1981a: 324). Insofern erfordert die kommunikative Reproduktion einer Disziplin als identifizierbarer Einheit die je neu aus der Perspektive der Gegenwart zu unternehmende Rekonstruktion ihrer Tradition. Selbstthematisierung des Systems im System ist nur mit Hilfe drastischer Vereinfachungen möglich. Was als Tradition jeweils präsent gehalten werden kann, unterliegt Beschränkungen. Man muß seligieren, sich an eine enge Auswahl von Theorien und Texten halten, die - als ,klassisch' etikettiert - verbindliche Anschlußstellen für die systemische Identitätsreflexion bilden.

Was aber heißt es, einen Text als Klassiker zu lesen? - Es „heißt gerade nicht: ihn historisch interpretieren. ... Man muß in der Analyse klassischer Texte den gegenwärtigen Stand des Faches zugrunde legen: das inzwischen gestiegene Auflösungsvermögen, die größere Tiefenschärfe der theoretischen und methodischen Gegenstandsprojektion.“ (Luhmann 1977:; 17f). Die Aneignung überlieferter Texte aus dem Vorverständnishorizont der Gegenwart wird damit zum Gebot erhoben. Wird es befolgt, dann ,geht die Rekonstruktion der Frage, aus der sich der Sinn eines Textes als Antwort versteht, in unser eigenes Fragen über" (Gadamer 1965: 356). Der Interpret versteht den Klassiker als Antwortversuch auf eine Frage, ein Problem, das sich ihm selbst ebenfalls stellt. Und insofern der Vorverständnishorizont als Horizont des Fraglichen zu bestimmen ist, auf das ein Text antwortet, findet „Horizontverschmelzung" statt zwischen dem Horizont des Interpreten und dem Horizont, aus dem der Text spricht (vgl. Gadamer 1965: 356f). Die Funktion der Wissenschaft, ihre Verpflichtung auf Erkenntniswachstum, macht es allerdings unwahrscheinlich, daß am Ende der Lektüre eines Klassikers auch die Übernahme seiner Antworten als uneingeschränkt gültige Problemlösungen steht. Die Aktualität eines klassischen Textes für eine wissenschaftliche Disziplin ist daran nicht gebunden. „Der Text bleibt aktuell, solange seine Problemstellung kontinuierbar ist. Er bleibt maßgebend in einem ambivalenten Sinne: Man kann an ihm ablesen, was zu leisten wäre; aber nicht mehr: wie es zu leisten ist" (Luhmann 1977: 18).

In der Identitätsreflexion wissenschaftlicher Disziplinen lebt Tradition in der beschriebenen Weise auch für die Systemtheorie fort. Und insofern Luhmann sich dieser Aufgabe mit den Mitteln der Interpretation klassischer Texte widmet, formuliert und befolgt er selbst Anforderungen (vgl. auch Luhmann 1981b), in denen er mit Gadamers Hermeneutik übereinkommt.

\section{Hermeneutik als Methode systemtheoretischer Forschung}

Hermeneutisches Verstehen als Methode der Systemtheorie - diese Verknüpfung mobilisiert Skepsis. Wie und wo ,versteht" die Systemtheorie im hermeneutischen Sinne dieses Begriffs? Verzichtet die Systemtheorie in ihrer ,Forschungspraxis ' de facto nicht gerade auf Verstehen zugunsten kategorialer Deduktion und der bloßen Subsumtion empirischer Bruchstücke unter ihre abstrakten Kategorien? - Wie auf diese Bedenken zu antworten ist, kann hier nur noch angedeutet werden mit Hilfe eines kurzen aber u.E. repräsentativen Beispiels aus Luhmanns Analysen zur „Gesellschaftsstruktur und Semantik“.

Dort untersucht Luhmann u.a. die Essais de Morale von Pierre Nicole (1671-1674) (Luhmann 1980: 109ff). Er zeichnet dabei nach, wie der Versuch, eine religiös begründete Interaktionsmoral zu entwerfen, sich in widersprüchlichen Anforderungen verfängt:

Die religiös begründete Forderung, anderen unter Verletzung ihrer Selbstliebe durch Aufklärung zur heilsnotwendigen Selbsterkenntnis zu verhelfen, kollidiert mit der gleichfalls religiösen Verpflichtung zur Wahrung des Friedens (vgl. Luhmann 1980: 111f). An diesem und weiteren Beispielen versucht Luhmann zu zeigen, wie die exzessive Strapazierung von Religion zur Lösung von Interaktionsproblemen in Paradoxien gerät, deren Bewältigung schließlich zur Ununterscheidbarkeit zwischen religiös empfohlener und weltlicher Interaktionsmoral und damit zur Selbstmarginali- 
sierung der Religion für die Regelung der Interaktion führt. Diese Entwicklung wird analysiert als Beispiel für den generellen Trend zur Entlastung der Oberschichteninteraktion von funktionssystemspezifischen Anforderungen. Diesen Trend wiederum interpretiert Luhmann systemtheoretisch als „Reaktion auf zunehmende Differenzierung der Systembildungsebenen Gesellschaft und Interaktion" (a.a.O.: 126), die einhergeht mit der Ausdifferenzierung funktionsspezifischer Teilsysteme.

Deutlich wird hier, wie Luhmanns Analyse Problemstufen unterschiedlicher Abstraktionsniveaus miteinander verbindet und dadurch hermeneutische Einzelfallanalyse und theoretische Generalisierung systematisch miteinander verknüpft. ${ }^{35} \mathrm{Da}-$ bei verfährt er offensichtlich theoriegeleitet, mit spezifisch gerichtetem Interesse, ohne sich zu binden an die Mitteilungsintentionen der untersuchten Autoren oder den Vorverständnishorizont der Zeitgenossen. Ihn interessiert, wie sich in der Semantik der Interaktion die kulturellen Voraussetzungen für die Umstellung der Differenzierung des Gesellschaftssystems auf den Primat funktionaler Differenzierung abzeichnen. Im Ausgang von diesem theoriegenerierten Problem erfüllt sich die Applikation auf den Vorverständnishorizont des Interpreten und eine daraus entspringende Frage, die für Gadamer konstitutiv für jedes Verstehen ist. Wiewohl historisch gerichtet, ist Luhmanns Frage nicht selbst historisch (in dem Sinne, wie es etwa für uns die Frage wäre, woran man früher Hexen glaubte erkennen zu können), sondern eine Frage, die sich für den Interpreten selbst wirklich erhebt. ${ }^{36} \mathrm{DaB}$ dieses Vorverständnis dem Kontext

35 Hier ist zu erinnern an die oben festgestellte Äquivalenz der hermeneutischen Figur von Frage und Antwort und der Unterscheidung von Problem und Problemlösung. Durch rekursive Anwendung kann diese Unterscheidung in einer Hierarchie von Problemstufen entfaltet und die Analyse uber verschiedene Stufen der Abstraktion geführt werden. Dadurch wird es möglich, die Leistungen hermeneutischer Interpretation und funktionaler Analyse miteinander zu verknüpfen. Vgl. dazu ausführlich Schneider 1991: Kap. 8 und 10 sowie Schneider 1992; zum Konzept der Problemstufenhierarchie vgl. Luhmann 1974: 18ff.

36 Insofern war es nur konsequent, daß Gadamer in einer Diskussion mit Luhmann, die im Dezember 1989 am Graduiertenkolleg in Siegen stattfand, dessen Untersuchungen zu Gesellschaftsstruktur und Semantik ohne zu zögern als Beispiele hermeneutischen Verstehens qualifizierte. Im übrigen war, wie einer wissenschaftlichen Theorie entstammt, ist dabei sekundär. ${ }^{37}$

Zielt aber eine solche Frage nicht über den Rahmen des hermeneutisch Zugänglichen hinaus? $\mathrm{Zu}$ diesem Ergebnis kommt nur, wer der Meinung ist, daß die Hermeneutik auf das Erkennen von bereits Erkanntem und in der kulturellen Überlieferung Niedergelegtem beschränkt ist. ${ }^{38}$ Gegen diese Auffassung hat sich Gadamer in der Diskussion mit Habermas ausdrücklich gewandt. ${ }^{39} \mathrm{Sie}$ gründet letztlich in der „romantischen“ Identifikation von Sinn mit subjektiv gemeintem Sinn, von der sich Gadamers Hermeneutik und Luhmanns Systemtheorie gleichermaßen distanzieren.

\section{Literatur}

Apel, K.O., 1971: Szientistik, Hermeneutik, Ideologiekritik. Entwurf einer Wissenschaftslehre in Erkenntnisanthropologischer Sicht. S. 7-44 in: J. Habermas/ D. Henrich/J. Taubes (Hrsg.), Hermeneutik und Ideologiekritik. Frankfurt/M.: Suhrkamp.

Bateson, G., 1983: Ökologie des Geistes. Frankfurt/M.: Suhrkamp.

Betti, E., 1962: Die Hermeneutik als allgemeine Methodik der Geisteswissenschaften. Tübingen: Mohr.

Blumenberg, H., 1966: Die Legitimität der Neuzeit. Frankfurt/M.: Suhrkamp.

hier eher anekdotisch anzumerken ist, diese Diskussion von einem merkwürdigen Mangel an kontroversem Stoff zwischen den ,Kontrahenten' gekennzeichnet, der freilich vor dem Hintergrund unseres Vergleichs wenig überraschend erscheint.

37 Gadamer (1965: 181, Fußn. 1) notiert für die Interpretation von Kunstwerken, anders als etwa die Selbstinterpretation eines Schriftstellers könne „.. . die ,Theorie', z. B. der Musik oder der Poetik und Redekunst, sehr wohl ein legitimer Kanon der Auslegung sein".

38 In diesem Sinne stellt Habermas (1971: 52) fest, „Die Hermeneutik stößt gleichsam von innen an Wände des Traditionszusammenhangs" und warnt vor einem „Idealismus der Sprachlichkeit", der ,gesellschaftliche Prozesse ganz zu kultureller Überlieferung sublimiert" (a.a.O.: 55).

39 Gadamer (1970: 70) hält dem entgegen, „Es verkürzt die Universalität der hermeneutischen Dimension, wenn ein Bereich des verständlichen Sinnes (,kulturelle Überlieferung') gegen andere, lediglich als Realfaktoren erkennbare Determinanten der gesellschaftlichen Wirklichkeit abgegrenzt wird", und etwas später mit einer Metapher (a.a.O.: 71f) ,im Spiegel der Sprache reflektiert sich vielmehr alles, was ist." 
Böhler, D., 1981: Philosophische Hermeneutik und hermeneutische Methode. S. 483-511 in: M. Fuhrmann et al. (s.u.).

Chladenius, J.M., 1742: Einleitung zur richtigen Auslegung vernünftiger Reden und Schriften. Abgedruckt 1976. S. 69-79 in: H.-G. Gadamer/G. Boehm, Seminar: Philosophische Hermeneutik. Frankfurt/M.: Suhrkamp.

Collingwood, R.G., 1955: Denken. Eine Autobiographie. Eingeleitet von Hans-Georg Gadamer. Stuttgart: Koehler.

Collingwood, R.G., 1957: An Essay on Metaphysics. Oxford: Clarendon.

Frank, M., 1985: Das individuelle Allgemeine. Textstrukturierung und Textinterpretation nach Schleiermacher. Frankfurt/M.: Suhrkamp.

Frank, M., 1990: Das Sagbare und das Unsagbare. Studien zur deutsch-französischen Hermeneutik und Texttheorie. Erweiterte Neuausgabe. Frankfurt/M.: Suhrkamp.

Fuchs, P., 1992: Die Erreichbarkeit der Gesellschaft: Zur Konstruktion und Imagination gesellschaftlicher Einheit. Frankfurt/M.: Suhrkamp.

Fuhrmann, M./JauB, H.R./Pannenberg, W. (Hrsg.), 1981: Text und Applikation. Theologie, Jurisprudenz und Literaturwissenschaft im hermeneutischen Gespräch. Poetik und Hermeneutik IX. München: Fink.

Gadamer, H.G., 1965: Wahrheit und Methode. Grundzüge einer philosophischen Hermeneutik. 2., erw. Auflage. Tübingen: Mohr.

Gadamer, H.G., 1971: Rhetorik, Hermeneutik und Ideologiekritik. Metakritische Erörterungen zu ,Wahrheit und Methode‘. S. 57-82 in: J. Habermas/ D. Henrich/J. Taubes (Hrsg.), Hermeneutik und Ideologiekritik. Frankfurt/M.: Suhrkamp.

Gadamer, H.G., 1974: Art. Hermeneutik. Sp. 10611073 in: J. Ritter (Hrsg.), Historisches Wörterbuch der Philosophie. Bd. 2. Basel/Stuttgart: Schwabe.

Gadamer, H.G., 1984: Text und Interpretation. S. 24-55 in: P. Forget (Hrsg.), Text und Interpretation. München: Fink.

Gadamer, H.G., 1986: Gesammelte Werke. Bd. 2. Wahrheit und Methode. Ergänzungen und Register. Tübingen: Mohr.

Gadamer, H.G./Boehm, G. (Hrsg.), 1976: Seminar: Philosophische Hermeneutik. Frankfurt/M. Suhrkamp.

Gadamer, H.G./Boehm, G. (Hrsg.), 1978: Seminar: Die Hermeneutik und die Wissenschaften. Frankfurt/ M.: Suhrkamp.

Habermas, J., 1970: Zur Logik der Sozialwissenschaften. Frankfurt/M.: Suhrkamp.

Habermas, J., 1971: Der Universalitätsanspruch der Hermeneutik. S. 120-159 in: J. Habermas/D. Henrich/J. Taubes (Hrsg.), Hermeneutik und Ideologiekritik. Frankfurt/M.: Suhrkamp.

Habermas, J., 1971a: Zu Gadamers, Wahrheit und Methode'. S. 45-56 in: J. Habermas/D. Henrich/J. Taubes (Hrsg.), Hermeneutik und Ideologiekritik. Frankfurt/M.: Suhrkamp.
Habermas, J., 1973: Erkenntnis und Interesse. 3. Auflage mit einem neuen Nachwort. Frankfurt/M.: Suhrkamp.

Habermas, J., 1981: Theorie des kommunikativen Handelns. 2 Bde. Frankfurt/M.: Suhrkamp.

Herzog, R., 1981: Vergleichende Bemerkungen zur theologischen und juristischen Applikation (am Beispiel zweier Auslegungen von 2. Sam. 11). S. 367-393 in: M. Fuhrmann et al. (s.o).

Herzog, R., 1981a: Zum Verhältnis von Norm und Narrativität in den applikativen Hermeneutiken. S. 435455 in: M. Fuhrmann et al. (s.o.)

Hirsch, E.D., 1972: Prinzipien der Interpretation. München.

JauB, H.R., 1981: Zur Abgrenzung und Bestimmung einer literarischen Hermeneutik. S. 459-481 in: $M$. Fuhrmann et al. (s.o.).

Kneer, G./Nassehi, A., 1991: Verstehen des Verstehens. Eine systemtheoretische Revision der Hermeneutik. Zeitschrift für Soziologie 20: 341-356.

Luhmann, N., 1974: Soziologische Aufklärung. Bd. 1. Aufsätze zur Theorie sozialer Systeme. Opladen: Westdeutscher.

Luhmann, N., 1977: Arbeitsteilung und Moral. Durkheims Theorie. S. 17-35. Einleitung zu: E. Durkheim, Über die Teilung der sozialen Arbeit. Frankfurt/M.: Suhrkamp.

Luhmann, N., 1980: Gesellschaftsstruktur und Semantik. Studien zur Wissenssoziologie der modernen Gesellschaft. Bd. 1. Frankfurt/M.: Suhrkamp.

Luhmann, N., 1981: Ist Kunst codierbar? S. 245-266 in: N. Luhmann, Soziologische Aufklärung. Bd. 3. Soziales System, Gesellschaft, Organisation. Opladen: Westdeutscher.

Luhmann, N., 1981a: Theoretische und praktische Probleme anwendungsbezogener Sozialwissenschaften. S. 321-334 in: N. Luhmann, Soziologische Aufklärung. Bd. 3. Opladen: Westdeutscher.

Luhmann, N., 1981b: Wie ist soziale Ordnung möglich? S. 195-285 in: Gesellschaftsstruktur und Semantik. Bd. 2. Studien zur Wissenssoziologie der modernen Gesellschaft. Frankfurt/M.: Suhrkamp.

Luhmann, N., 1984: Soziale Systeme. Grundriß einer allgemeinen Theorie. Frankfurt/M.: Suhrkamp.

Luhmann, N., 1986: Systeme verstehen Systeme. S. 72117 in: N. Luhmann/K.E. Schorr (Hrsg.), Zwischen Intransparenz und Verstehen. Fragen an die Pädagogik. Frankfurt/M.: Suhrkamp.

Luhmann, N., 1986a: Das Kunstwerk und die Selbstreproduktion der Kunst. S. 620-672 in: H.U. Gumbrecht/K.L. Pfeiffer (Hrsg.), Stil. Geschichten und Funktionen eines kulturwissenschaftlichen Diskurselementes. Frankfurt/M.: Suhrkamp.

Luhmann, N., 1987: „Distinctions directrices“. Über Codierung von Semantiken und Systemen. S. 13-31 in: N. Luhmann, Soziologische Aufklärung. Bd. 4. Beiträge zur funktionalen Differenzierung der Gesellschaft. Opladen: Westdeutscher.

Luhmann, N., 1988: Wie ist Bewußtsein an Kommunikation beteiligt? S. 884-905 in: H.U. Gumbrecht/ 
K.L. Pfeiffer (Hrsg.), Materialität der Kommunikation. Frankfurt/M.: Suhrkamp.

Luhmann, N., 1989: Gesellschaftsstruktur und Semantik. Studien zur Wissenssoziologie der modernen Gesellschaft. Bd. 3. Frankfurt/M.: Suhrkamp.

Luhmann, N., 1990: Die Wissenschaft der Gesellschaft. Frankfurt/M.: Suhrkamp.

Luhmann, N., 1990a: Das Erkenntnisprogramm des Konstruktivismus und die unbekannt bleibende Realität. S. 31-58 in: N. Luhmann, Soziologische Aufklärung. Bd. 5. Konstruktivistische Perspektiven. Opladen: Westdeutscher.

Luhmann, N., 1990b: Identität - was oder wie? S. 14-30 itı: N. Luhmann, Soziologische Aufklärung. Bd. 5. Konstruktivistische Perspektiven. Opladen: Westdeutscher.

Luhmann, N., 1990c: Weltkunst. S. 7-45 in: N. Luhmann, F.D. Bunsen, D. Baecker, Unbeobachtbare Welt. Bielefeld: Haux.

Luhmann, N., 1990d: Sthenographie. S. 119-137 in: N. Luhmann/H. Maturana/M. Namiki/V. Redder/F. Varela, Beobachter. Konvergenz der Erkenntnistheorien? München: Fink

Luhmann, N., 1991: Am Ende der kritischen Soziologie. Zeitschrift für Soziologie 20: 147-152.

Luhmann, N., 1991a: Wie lassen sich latente Strukturen beobachten? S. 61-74 in: P. Watzlawick/P. Krieg (Hrsg.), Das Auge des Beobachters. Beiträge zum Konstruktivismus. Festschrift für Heinz von Foerster. München: Piper.

Luhmann, N./Fuchs, P., 1989: Reden und Schweigen. Frankfurt/M.: Suhrkamp.

Madison, G.B., 1978: Eine Kritik an Hirschs Begriff der „Richtigkeit“. S. 393-425 in: H.G. Gadamer/G. Boehm (Hrsg.), Seminar: Die Hermeneutik und die Wissenschaften. Frankfurt/M.: Suhrkamp.

Marquard, O., 1981: Frage nach der Frage, auf die die Hermeneutik die Antwort ist. S. 115-146 in: O. Marquard, Abschied vom Prinzipiellen. Stuttgart: Reclam.

Oevermann, U./Allert, T./Konau, E./Krambeck, J., 1979: Die Methodologie einer ,objektiven Herme- neutik' und ihre allgemeine forschungslogische $\mathrm{Be}$ deutung in den Sozialwissenschaften. S. 352-434 in: H.G. Soeffner (Hrsg.), Interpretative Verfahren in den Sozial- und Textwissenschaften. Stuttgart: Metzler.

Oevermann, U., 1986: Kontroversen über sinnverstehende Soziologie. Einige wiederkehrende Probleme und Mißverständnisse in der Rezeption der ,objektiven Hermeneutik'. S. 19-83 in: S. Aufenanger/M. Lenssen (Hrsg.), Handlung und Sinnstruktur. Bedeutung und Anwendung der objektiven Hermeneutik. München: Kindt.

Ricoer, P., 1978: Der Text als Modell: hermeneutisches Verstehen. S. 83-117 in: H.G. Gadamer/G. Boehm (Hrsg.), Seminar: Die Hermeneutik und die Wissenschaften. Frankfurt/M.: Suhrkamp.

Schleiermacher, F.D.E., 1977: Hermeneutik und Kritik. Herausgegeben und eingeleitet von Manfred Frank. Frankfurt/M.: Suhrkamp.

Schneider, W.L., 1991: Objektives Verstehen. Rekonstruktion eines Paradigmas: Gadamer-Popper-Toulmin-Luhmann. Opladen: Westdeutscher.

Schneider, W.L., 1991a: Objektive Hermeneutik und Systemtheorie. Theoretische und methodologische Konvergenzen. (Beitrag zum Kolloquium „Sachliche und methodische Konvergenzen in der interaktionsanalytischen Forschung" vom 17.-19. Okt. 1991 in Konstanz).

Schneider, W.L., 1992: Hermeneutische Einzelfallrekonstruktion und funktionalanalytische Theoriebildung. Ein Versuch ihrer Verknüpfung, dargestellt am Beispiel der Interpretation eines Interviewprotokolls. S. $168-215$ in: J.H.P. Hoffmeyer-Zlotnik (Hrsg.), Analyse verbaler Daten. Über den Umgang mit qualitativen Daten. Opladen: Westdeutscher.

Schönke, A./Schröder, H., 1988: Strafgesetzbuch. Kommentar. 23. Auflage. München: Beck.

Soeffner, H.G., 1989: Auslegung des Alltags - Alltag der Auslegung. Zur wissenssoziologischen Konzeption einer sozialwissenschaftlichen Hermeneutik. Frankfurt/M.: Suhrkamp. 